\title{
TRACKING THE SOLAR CYCLE THROUGH IBEX OBSERVATIONS OF ENERGETIC NEUTRAL ATOM FLUX VARIATIONS AT THE HELIOSPHERIC POLES
}

\author{
D. B. Reisenfeld ${ }^{1}$, M. Bzowsir ${ }^{2}$, H. O. Funsten $^{3}$, S. A. Fuselier ${ }^{4,5}$, A. Galli ${ }^{6}$, P. H. Janzen ${ }^{1}$, \\ N. Karna ${ }^{7,8}$, M. A. KubiaK ${ }^{2}$, D. J. McComas ${ }^{9}$, N. A. Schwadron ${ }^{10}$, and J. M. SoKó ${ }^{2}$ \\ ${ }^{1}$ University of Montana, Missoula, MT 59812, USA; dan.reisenfeld@umontana.edu, paul.janzen@umontana.edu \\ 2 Space Research Centre of the Polish Academy of Sciences, (CBK PAN), Bartycka 18A, 00-716, Warsaw, Poland; \\ bzowski@cbk.waw.pl,mkubiak@cbk.waw.pl,jsokol@cbk.waw.pl \\ ${ }^{3}$ Los Alamos National Laboratory, Los Alamos, NM 87545, USA; hfunsten@lanl.gov \\ ${ }^{4}$ Southwest Research Institute, San Antonio, TX 78228, USA; sfuselier@swri.edu \\ ${ }^{5}$ University of Texas at San Antonio, San Antonio, TX 78228, USA \\ ${ }^{6}$ Physics Institute, University of Bern, Bern 3012, Switzerland; andre.galli@space.unibe.ch \\ George Mason University, Fairfax, VA 22306, USA; nkarna@masonlive.gmu.edu \\ ${ }^{8}$ NASA Goddard Space Flight Center, Greenbelt, MD, USA \\ ${ }^{9}$ Princeton University, Peyton Hall, Princeton, NJ 08544, USA; dmccomas@ princeton.edu \\ ${ }^{10}$ University of New Hampshire, Space Science Center, Durham, NH 03824, USA; n.schwadron@unh.edu \\ Received 2016 July 17; revised 2016 October 27; accepted 2016 October 29; published 2016 December 21
}

\begin{abstract}
With seven years of Interstellar Boundary Explorer (IBEX) observations, from 2009 to 2015, we can now trace the time evolution of heliospheric energetic neutral atoms (ENAs) through over half a solar cycle. At the north and south ecliptic poles, the spacecraft attitude allows for continuous coverage of the ENA flux; thus, signal from these regions has much higher statistical accuracy and time resolution than anywhere else in the sky. By comparing the solar wind dynamic pressure measured at 1 au with the heliosheath plasma pressure derived from the observed ENA fluxes, we show that the heliosheath pressure measured at the poles correlates well with the solar cycle. The analysis requires time-shifting the ENA measurements to account for the travel time out and back from the heliosheath, which allows us to estimate the scale size of the heliosphere in the polar directions. We arrive at an estimated distance to the center of the ENA source region in the north of 220 au and in the south a distance of 190 au. We also find a good correlation between the solar cycle and the ENA energy spectra at the poles. In particular, the ENA flux for the highest $I B E X$ energy channel $(4.3 \mathrm{keV})$ is quite closely correlated with the areas of the polar coronal holes, in both the north and south, consistent with the notion that polar ENAs at this energy originate from pickup ions of the very high speed wind $\left(\sim 700 \mathrm{~km} \mathrm{~s}^{-1}\right)$ that emanates from polar coronal holes.
\end{abstract}

Key words: ISM: general - magnetohydrodynamics (MHD) - plasmas - solar wind - Sun: heliosphere

\section{INTRODUCTION}

The Interstellar Boundary Explorer (IBEX) mission (McComas et al. 2009a) has been collecting energetic neutral atoms (ENAs) of heliospheric origin for seven years. Over this time period, there has been considerable temporal evolution of both the ENA flux intensity and energy distribution. Because the ENA observations span a substantial portion of a solar cycle, it is now possible to carry out quantitative studies of the correlation between changes in the ENA flux and solar wind (SW) parameters that evolve with the solar cycle.

A number of studies have reported on the variation of the ENA flux observed by IBEX (e.g., McComas et al. 2010, 2012, 2014; Reisenfeld et al. 2012). Most recently, McComas et al. (2014) showed that during the first four years of observations, from 2009 to 2012, the ENA flux generally declined, by as much as $40 \%$, across most of the sky, but that between 2012 and 2013, the flux decline began to level off across much of the sky. Exceptions are in the direction of the heliotail, where the flux decline continued at all energies, and at high latitudes, where the flux measured in the two highest IBEX energy channels $(2.7$ and $4.3 \mathrm{keV})$ also continued to decline. The magnitude of the variation is asymmetric in latitude: by 2013 the flux at high southern latitudes dropped by almost a factor of two relative to 2009 , whereas the flux at northern latitudes dropped by as much as a factor of three (see Figure 20, McComas et al. 2014). The IBEX Ribbon, the region of enhanced ENA emissions circumscribing the sky (McComas et al. 2009b; Funsten et al. 2009b; Fuselier et al. 2009a; Schwadron et al. 2009; 2011), shows time variations generally consistent with the globally distributed flux.

The ENA energy spectra also change with time. The energy spectrum is typically characterized as a power law, $j(E) \propto E^{-\gamma}$ (where $j$ is the ENA flux, $E$ is the energy, and $\gamma$ is the spectral index; Funsten et al. 2009b; Dayeh et al. 2011, 2012; Schwadron et al. 2011, 2014; McComas et al. 2014; Desai et al. 2015). Averaged over time, the energy spectra show a strong latitudinal dependence, being harder at high latitudes $(\gamma \lesssim 1.5)$ and softer at low latitudes $(1.5 \lesssim \gamma \lesssim 2)$. This is seen as a consequence of the higher SW speed at high latitudes in the periods around solar minimum (e.g., Schwadron et al. 2011; Dayeh et al. 2012). McComas et al. (2014) also showed that, in the tailward hemisphere, the spectral index has larger values $(\gamma>2.5)$ in two broad regions at low to mid latitudes, associated with the side lobes of a large heliotail structure centered in nearly the downwind direction.

The time evolution of the spectral index has been studied by McComas et al. (2012, 2014) and Dayeh et al. (2012). McComas et al. (2014) find both nose-tail and north-south asymmetries in the evolution of the spectral index over time. Between 2009 and 2013, the spectral index generally increased in the upwind (toward the nose) hemisphere and decreased in the downwind hemisphere. Additionally, the spectral index on the upwind side shows a substantial north-south asymmetry, 
with a largely increasing spectral slope in the north and a weakly decreasing slope in the south.

These observational studies have been complemented by theoretical work describing how the heliosphere may evolve with the solar cycle. Work on this subject dates to Belcher et al. (1993), who used a one-dimensional kinematic model to study the effects of SW ram pressure on the termination shock in an effort to explain Pioneer 10 and 11 (Barnes 1990) and Voyager 2 (Lazarus \& McNutt 1990) observations of fluctuations in the SW ram pressure. Recent models (e.g., Izmodenov et al. 2008; Washimi et al. 2011; Pogorelov et al. 2013) are now fully three-dimensional and use as constraints data provided by the Voyager spacecraft as they journey through the heliosheath. Particularly relevant to our work, recent efforts have gone into modeling the time variation in the 1 au ENA flux based on realistic solar cycle parameters (e.g., Fahr \& Scherer 2004; Sternal et al. 2008; Siewert et al. 2014; Zirnstein et al. 2015). See Zirnstein et al. (2015) for a comprehensive summary of the theoretical body of research on the influence of the solar cycle on the heliosphere.

Returning to the observational studies, most of the works cited above have a temporal resolution of 12 months. This is because, although it takes the sensors on the IBEX spacecraft six months to observe the complete sky, adjacent six-month sky maps at a given energy are not identical, due to the motion of the spacecraft in the Sun's inertial frame. Only sky maps with annual separations measure the same incident ENA energies. However, it is possible to study time variations on timescales shorter than even six months because two regions of the sky, the ecliptic poles, are observed nearly continuously by $I B E X$. Continuous observation also means the statistical uncertainties for the polar fluxes are substantially smaller than anywhere else in the sky, and thus time variations can be assessed with greater statistical accuracy.

The purpose of this paper is to understand what aspects of the solar wind most directly drive temporal changes in the observed heliospheric ENA fluxes. Here we demonstrate that the time variation in the ENA flux is driven primarily by changes in the dynamic pressure $\left(\rho v^{2}\right)$ that the SW exerts on the inner boundary of the heliosheath at the termination shock (TS). We also show that temporal variations in the outgoing solar wind's energy spectrum is preserved beyond the TS and leaves a measurable imprint on the ENA flux.

We utilize a method previously developed (Reisenfeld et al. 2012, hereafter R2012) to correlate the line-of-sight (LOS)-integrated heliosheath plasma pressure (derived from the ENA flux) with the outbound SW dynamic pressure observed at 1 au two to five years prior. It is valid to compare the polar ENA flux to the ecliptic 1 au SW dynamic pressure because the dynamic pressure appears to be independent of heliolatitude (McComas et al. 2008). The R2012 study was performed using the first two years of IBEX-Hi data for the energy range $0.5-6 \mathrm{keV}$. During this period, the ENA flux steadily decreased at all energies, and this trend correlated well with the steady decline in SW dynamic pressure observed at 1 au between 2005 and 2009, corresponding to the declining phase of solar cycle 23 (SC23). The method detailed in R2012 and summarized later in this paper (see Section 4.3.2) was used to infer the distances to the TS and the thickness of the inner heliosheath (IHS) in the direction of the ecliptic poles. R2012 estimated TS distances of $110 \mathrm{au}$ and $134 \mathrm{au}$ at the south and north poles, respectively, and corresponding IHS thicknesses of 55 and $82 \mathrm{au}$.

Because the time period of the R2012 study was relatively brief compared to a complete solar cycle, we considered the correlations and resulting distance determinations to be preliminary. The present study extends the data set an additional five years, taking the analysis through over half a solar cycle from 2009 through the end of 2015. During this period, the Sun completed SC23 and began solar cycle 24 (SC24), evolving from solar minimum to solar maximum conditions, with the observed 1 au SW dynamic pressure passing through a minimum. This allows for a betterconstrained correlation between ENA flux and dynamic pressure than was made in R2012 and, in principle, a more accurate estimation of the dimensions of the heliosphere.

In Section 2, we describe the method of data collection and analysis and discuss improvements in the method of background removal from the data since the first study. In Section 3, we present the north and south ecliptic pole ENA fluxes at different energies as a function of time, from 0.2 to $6 \mathrm{keV}$, using data from both IBEX sensors (IBEX-Hi and IBEX-Lo). We also present the evolution of the polar ENA energy spectrum over time. In Section 4 we discuss the mechanisms by which SW variations couple to the ENA flux and show evidence for these mechanisms. Section 5 summarizes our results and discusses future work.

\section{DATA ANALYSIS}

\subsection{IBEX Data Collection}

The primary data set used for this analysis consists of polar ENA fluxes collected by IBEX over the course of seven years, between 2008 December 25 and 2015 December 24 (orbits 11-310). The full details of IBEX data acquisition and instrument pointing germane to the observations of ENAs incident from the ecliptic poles are described in R2012. Here we summarize the IBEX data collection process, pointing out conditions that have changed since the first study (R2012).

There are two sensors on IBEX capable of detecting neutral atoms: IBEX-Lo, with an energy range of $10 \mathrm{eV}$ to $2 \mathrm{keV}$ (Fuselier et al. 2009b), and IBEX-Hi, with an energy range of $500 \mathrm{eV}$ to $6 \mathrm{keV}$ (Funsten et al. 2009a). Our analysis is based primarily on observations from the IBEX-Hi sensor, although in Section 3.3 we discuss the detection of polar ENAs by $I B E X$-Lo. IBEX is a Sun-pointing, spin-stabilized spacecraft, rotating at $\sim 4 \mathrm{rpm}$. The $I B E X-\mathrm{Hi}$ sensor has a roughly circular instantaneous field of view that is 6.5 (FWHM) wide, viewed perpendicular to the spacecraft spin axis. IBEX-Hi is designed to step through six discrete energy settings of its electrostatic analyzer (ESA) with an energy resolution of $\Delta E / E \sim 65 \%$ (FWHM)

From the beginning of the science mission (2008 December 25) through orbit 183 in 2011 October, IBEX-Hi collected ENAs at six logarithmically spaced energy steps. Beginning in orbit 184, the sequence of six energy steps was modified from a sequence of ESA 1-2-3-4-5-6 to ESA 2-3-3-4-5-6. ESA 1, the lowest-energy step (center energy $\sim 450 \mathrm{eV}$ ), which often had an elevated background, was dropped in favor of doubling the sampling of ESA $3(\sim 1.1 \mathrm{keV})$, where the IBEX Ribbon is most pronounced. The data reported here are from ESAs 2 through 6 . 
Another modification made to the operation of IBEX since the first study was the raising of the $I B E X$ orbital perigee from $\sim 3 R_{\mathrm{E}}$ to $\sim 8 R_{\mathrm{E}}$ during orbits 128 and 129 (2011 June), which changed the orbital period from $\sim 7.5$ to $\sim 9.1$ days, placing $I B E X$ in a long-term stable lunar-synchronous orbit with apogee remaining at $\sim 50 R_{\mathrm{E}}$ (McComas et al. 2011). The increased orbital period necessitated a modification to the repointing operations to keep the spacecraft Sun-pointing: starting with orbit 130 , the spacecraft is repointed by $\sim 4.5$ both near perigee and apogee rather than just once per orbit by $\sim 7^{\circ} .5$ near perigee, as was done previously.

The data products used for this study are the IBEX-Hi ENA histograms (see Funsten et al. 2009a), which divide the $360^{\circ} \times 6.5$ swath of sky viewed during a given orbit into 60 histogram bins separated by $6^{\circ}$. To collect counts from the ecliptic poles, we must account for the aberration of the incidence direction of polar ENAs that is due to the $30 \mathrm{~km} \mathrm{~s}^{-1}$ motion of the Earth (and thus IBEX) about the Sun. True polar ENAs arrive at $I B E X$ from a slightly prograde direction. For $I B E X-\mathrm{Hi}$, this effect is small, amounting to a shift ranging from $2^{\circ}$ to 4.5 , depending on the ESA setting. We adjust for the aberration by taking an appropriately weighted average of the histogram bin looking directly at a given ecliptic pole and the adjacent bin centered $6^{\circ}$ in the prograde direction. Note that no energy correction is needed as the motion of the Earth frame is transverse to the ecliptic pole directions.

\subsection{Backgrounds}

Because this study is concerned primarily with changes in the ENA flux, it is worth briefly explaining how the IBEX-Hi data are validated and corrected for local backgrounds that could introduce apparent time variability into the data if not taken into account. As part of routine IBEX-Hi data processing, data from each orbit's science collection period are culled of segments not usable for heliospheric science. One source of such background occurs when protons strike the IBEX-Hi entrance collimator and are neutralized. Such neutrals are then detectable if they are within the energy passband of the sensor. Time periods are therefore removed when the SW flow direction is sufficiently nonradial or the temperature is hot enough that protons are deflected onto the collimator, or when IBEX passes through the magnetosheath and the sensor is overwhelmed by the hotter magnetosheath ions. Periods are also removed when IBEX directly views ENAs of magnetospheric or lunar origin or when the energetic particle flux is particularly high.

Two other background sources are always present and thus must be quantified and subtracted from the data: the penetrating background due to cosmic radiation, and the "ion gun" background associated with hot SW electrons. These are discussed in detail in Appendices A and D, respectively, of McComas et al. (2014) and summarized briefly here.

Penetrating radiation background, the more significant of the two, appears in the qualified triple-coincidence count rate, which is the cleanest $I B E X-\mathrm{Hi}$ data product and the one used in the vast majority of $I B E X$ studies. The modifier "qualified" refers to the order in which the channel electron multipliers (CEMs) in the IBEX-Hi detector section are triggered. (See Funsten et al. (2009a) for a complete description of the $I B E X$ $\mathrm{Hi}$ data products and their method of generation.) The penetrating background rate over the course of the first 6.5 years of the mission has ranged over about $0.03-0.06 \mathrm{~s}^{-1}$, comparable to the magnitude of the heliospheric signal. Fortunately, penetrating radiation also creates "unqualified" triples, whereas true ENAs generate very few of these; thus, the unqualified triples rate can be monitored and, along with periodic on-orbit background monitoring tests, used to correct the qualified triples rate. The penetrating radiation contribution to the qualified triples can be very well characterized, having an uncertainty of only $2 \%-3 \%$.

The second ubiquitous background, the ion-gun background, is generated from ambient neutrals, such as desorbed or outgassed molecules in the $I B E X-\mathrm{Hi}$ instrument that become ionized within the positive collimator region of IBEX-Hi. From this location, they can be accelerated into the entrance conversion foil and masquerade as ENAs from outside the instrument (Wurz et al. 2009). Ionization is principally due to higher energy SW electrons that get past the negative collimator, which are subsequently accelerated into the positive collimator. It is important to note that an ion-gun background shows up in qualified triples almost entirely in ESAs 1, 2, 5, and 6 but is negligible in ESAs 3 and 4 (McComas et al. 2014).

Initially, typical values of the ion-gun count rate ranged from $0.010 \mathrm{~s}^{-1}$ in ESA 5 to $0.017 \mathrm{~s}^{-1}$ in ESA 6. After a period of testing, it was determined that it would be possible to operate the negative collimator at a higher voltage (from $-1170 \mathrm{~V}$ to $-1400 \mathrm{~V}$ ) such that the ion-gun background would be reduced by a factor of three or greater without increasing the background associated with deflected SW protons. Thus, from 2013 April onward, the negative collimator was operated at this higher voltage. As with the penetrating radiation background, the ion-gun background rate in the qualified triples can be determined by monitoring other IBEX-Hi coincidence data products. The method of correction is described in McComas et al. (2014).

\subsection{Detection Efficiency Correction}

The IBEX-Hi team has recently evaluated the sensor detection efficiency over the course of the mission to date, utilizing a technique developed by Funsten et al. (2005) for continuously monitoring detection efficiencies of triplecoincidence systems. The efficiency determination technique as applied to IBEX-Hi is described in Appendix B of McComas et al. (2014). In short, there has been a small but measurable change in the efficiency since the beginning of the mission, most of it occurring during the first year of operation. Between the beginning of science collection in late 2008 and the beginning of 2010, the sensor efficiency steadily dropped by $\sim 10 \%$, but then remained stable within measurement error until 2014. In the first half of 2014 (the acquisition period for Map 11 ), the detector CEM voltages were raised by $80 \mathrm{~V}$, which recovered about half of the early efficiency drop, raising the detection efficiency to $96 \%$ of what it was at the beginning of the mission.

The analysis presented in McComas et al. (2014) also showed a measurable drop in detection efficiency, but since the analysis was still preliminary, the efficiency of $I B E X$-Hi was taken as constant. Now that the analysis has been completed with a much higher degree of confidence, the polar ENA fluxes presented here have been adjusted for these efficiency changes. Accounting for this variation, particularly the $10 \%$ drop in the first year, gives rise to a noticeable difference between the fluxes presented here and those in R2012. This will be discussed further in Section 3.1. 


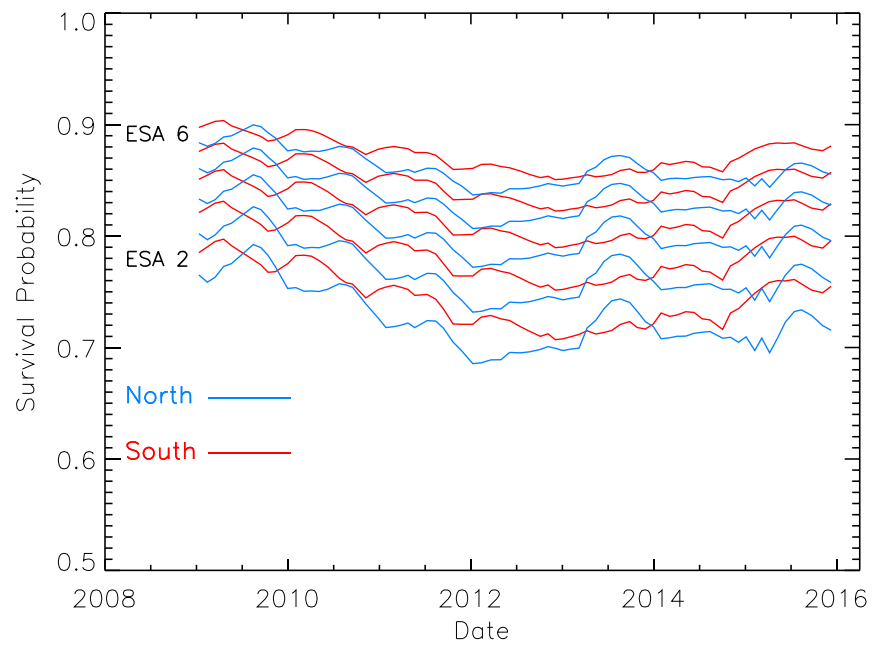

Figure 1. Survival probabilities for ENAs arriving at $I B E X$ from the north (blue) and south (red) ecliptic poles.

\subsection{ENA Survival Probability}

In order to isolate temporal changes to the outer heliosphere, we also correct the IBEX data for the ENA survival probability. A certain fraction of ENAs en route from the outer heliosphere to $1 \mathrm{au}$ are lost, either through ionization via photoionization or by charge exchange with outgoing SW ions and electrons, or by the effect of radiation pressure due to resonant absorption of solar Ly $\alpha$ radiation, an important effect especially during the maximum phase of solar activity. The loss effect is very important at lower energies $(\leqslant 0.15 \mathrm{keV})$, but at the ENA energies considered here $(0.5-6 \mathrm{keV})$, the losses are about 10-20\%. Survival probabilities of heliospheric ENAs are discussed by Bzowski (2008), and details of the survival probability calculations for the heliospheric ENAs observed by IBEX are presented in Appendix B of McComas et al. (2012) and further updated in Appendix B of McComas et al. (2014).

Survival probabilities for ENAs vary with the solar cycle, decreasing with the increase of solar activity. The variations of hydrogen ENAs are governed to a great extent by the SW and its heliolatitudinal structure because the charge-exchange reaction is the predominant loss process. Polar ENA survival probabilities are plotted in Figure 1 from the beginning of the mission through mid-2015. At the IBEX-Hi energies, the survival probabilities averaged over the mission range between $67 \%$ and $90 \%$, depending on energy; thus, extinction has a measurable but not dramatic effect on the observed fluxes of ENAs at $1 \mathrm{au}$. There is also a noticeable time variation in the extinction rates over the study period. The survival probabilities more-or-less steadily decline until 2012, tracking the increase in solar activity following the last solar minimum. Thereafter, during the years of peak solar activity, they stabilize. On top of the general trend rides an annual variation in the polar ENA extinction due to the north-south oscillation of the Earth (and hence $I B E X$ ) about the solar equatorial plane. When IBEX views through the solar equatorial plane, the observed ENAs have undergone greater extinction because the instrument is viewing through a greater distance to the heliospheric boundary than when viewing away from the equatorial plane. This explains why the annual variation has the opposite phase between the two poles.

The variation of the survival probability over the first seven years of the mission decreases with increasing energy, ranging from a variation of $\sim 15 \%$ between extremes for ESA 2 to $\sim 7 \%$ for ESA 6. The root-mean-square deviation over this period ranges from $\pm 3 \%$ for ESA 2 to $\pm 2 \%$ for ESA 6 . When considered in comparison to the statistical fluctuations of the IBEX ENA observations (see Section 3.1), evidence for variation of the ENA flux due to the survival probability should be noticeable on the scale of the entire mission, but not necessarily on shorter timescales.

\section{OBSERVATIONS}

\subsection{Polar Fluxes Observed by IBEX-Hi}

Figure 2 shows the differential ENA energy flux arriving at $I B E X$ from the north and south ecliptic poles. The data have been binned into roughly one-month intervals, and the error bars indicate the combined $1 \sigma$ Poisson counting statistic uncertainties of the measurements and the $1 \sigma$ uncertainties associated with the corrections for penetrating radiation, the hot electron-associated ion-gun background, and the time-varying detection efficiency. They do not, however, include any uncertainties associated with the survival probability correction or the presence of periods of unresolved deflected solar wind-associated background. Although it is possible that there is a small amount of deflected SW background remaining, it is likely no more than a 5\% contribution to ESAs 3-6. However, for ESA $2(710 \mathrm{eV})$, we estimate the level of deflected SW contamination at closer to $10 \%-15 \%$. ESA 2 is more susceptible to residual SW because (1) the SW background is the largest in ESA 2 and (2) the heliospheric signal rate is the lowest in ESA 2 due to the decreasing instrument sensitivity with decreasing energy (Funsten et al. 2009a). All residual SW background levels (even for ESA 2) are smaller than those present in the data shown in R2012 because, for that study, the ion-gun background had not yet been identified and removed.

Even though the polar signal is essentially continuous, we choose one-month binning not only because this gives reasonable statistics, but also because a time resolution shorter than this has no significance when considering observations of ENAs originating in the outer heliosphere. This is due to the rather large energy passband of the ESAs $(\Delta E / E \sim 65 \%)$, which results in significant time dispersion in the observation of ENAs originating beyond the TS $(\sim 100 \mathrm{au})$. For example, the ENA observed by the highest energy step, ESA 6, has a central energy of $4.3 \mathrm{keV}$ and an FWHM passband of $2.8 \mathrm{keV}$, which leads to a spread in ENA arrival times of two months for ENAs originating at $100 \mathrm{au}$. For ESA 2, the spread is six months (see Table 1, R2012). Thus, the shortest time resolution we expect is set by the two-month spread of ESA 6. For this reason, we present data at one-month time resolution in keeping with Nyquist-rate sampling practice. (It should also be mentioned that heliospheric ENAs do not originate from a point source but from a relatively thick emission region along the LOS, so the timescale for variation will likely be even longer than two months.)

To make long-term trends more evident, we plot in Figure 2 polar fluxes averaged over every six months. The statistical fluctuations are much reduced (by $\sim \sqrt{6}$ ), reflected by the smaller error bars. In addition, to complement the absolute flux plots, Figure 3 gives the relative flux variations. These points are the same six-month averages shown in Figure 2, but normalized to the earliest six-month interval of the mission. It 

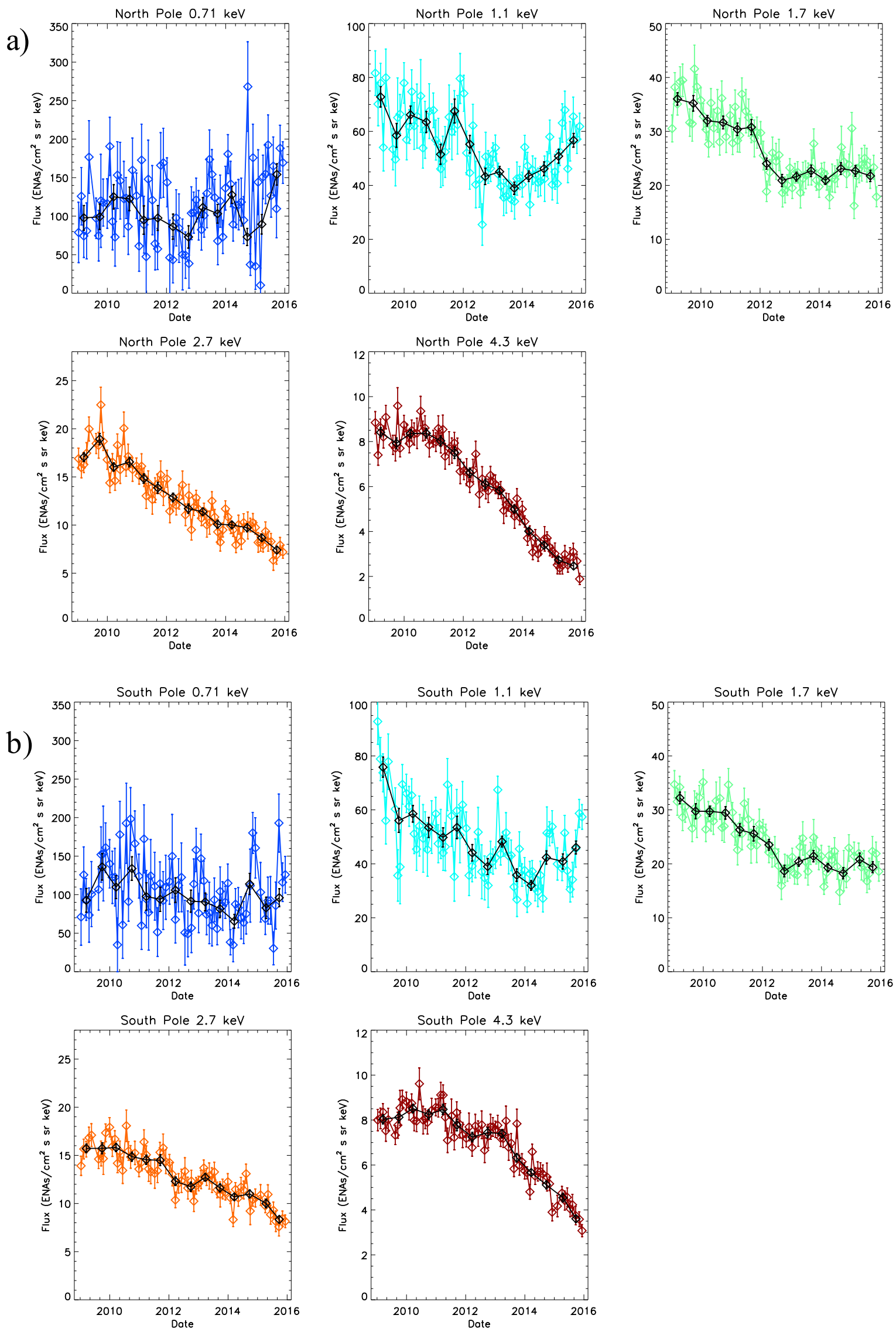

Figure 2. IBEX-Hi differential ENA energy fluxes at the (a) north and (b) south ecliptic poles. Each point represents roughly one month of data; error bars show $1 \sigma$ statistical uncertainty and uncertainties associated with subtraction of penetrating radiation background and SW electron-induced ion-gun background. Fluxes are corrected for ENA survival. The overlaid black points are six-month averages of the ENA flux. 

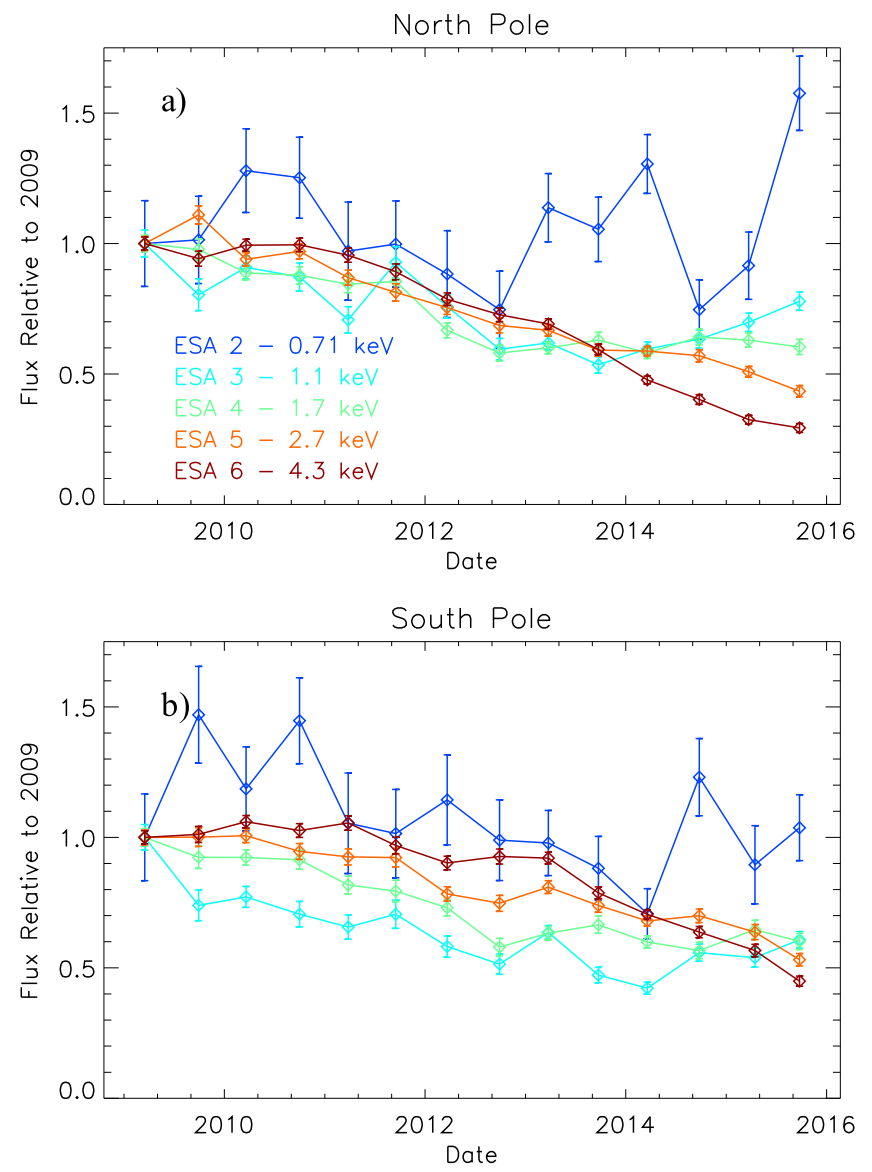

Figure 3. Six-month averages of $I B E X$-Hi differential ENA energy fluxes at the (a) north and (b) south ecliptic poles, normalized to the beginning of the mission. Data are the same as the black points shown in Figure 2.

is useful to compare Figure 3 to Figure 23 of McComas et al. (2014), which shows relative fluxes for various regions of the sky through 2013. The fractional changes in the north and south polar fluxes over time are consistent with those shown in their "nose/north pole" and "south pole/flanks" plots, respectively, even though these latter regions span a much larger range of latitudes.

Turning attention to the polar flux evolution in detail, over most of the mission all energy steps show periods of declining ENA flux, but the phasing varies. Beginning with ESAs 3 and 4 (deferring discussion on ESA 2 for the moment), at the start of the observation period they both exhibit decreasing ENA fluxes at both poles. In Figure 2, the monthly flux in ESA 3 drops by $\sim 50 \%$ by late 2013 , and the ESA 4 flux falls by $\sim 40 \%$ by the end of 2012. Thereafter, the flux in ESA 3 has clearly begun to increase, while the flux in ESA 4 stays fairly constant, with perhaps some sign of recovery in the north. The behavior at higher energies shows a markedly different evolution. The ESA 5 flux at both poles appears level for the first year, only starting to drop in 2010. It continues to decline for the rest of the observation period, by a factor of two. The flux in ESA 6 shows the most extreme variation. It is essentially flat for the first two years, even showing a slight rise at the south pole, and then begins to decline from 2011 onward with no clear sign yet of bottoming out. In the south, the ESA 6 flux has fallen by a factor of three, and, remarkably, in the north the flux has fallen by a full factor of four.
At first glance, the phasing of the flux variations between energy steps may appear counterintuitive. If the ENAs were all formed in a common source region, we would expect to see a particular pattern in the flux variation to appear at the highest energy first because of the faster travel time for such ENAs. This is not, however, the case. The leveling of the ENA flux appears first at low energies (ESAs 3 and 4) rather than high energies (ESAs 5 and 6). In fact, at the high energies the fluxes continue to fall. We return to this issue in Section 4.

\subsection{ESA 2}

The flux evolution in ESA 2 requires a separate discussion. Taking the one-month observations at face value, even if we ignore the most extreme points, the flux ranges over a factor of four to five, which seems unrealistic compared to what is observed at the other energies. Furthermore, because of the poor statistics, it is difficult to definitively identify clear trends over time. The six-month averaged data in Figures 2 and 3 damp most of the statistical variation and bring out the longterm trends. Averaging to this timescale is also justified because of the six-month time dispersion for this energy step for ENAs arriving from a point source beyond the TS. Thus any observed variations on timescales shorter than this must be due to statistical noise or a local background source, with the caveat that even with six-month averaging the uncertainty is still of the order of the magnitude of the variation.

From examination of Figure 3, we see that the curves for ESA 2 do not seem to be simply out of phase with the other energies, but they have a fundamentally different character, particularly for the north. This raises the question of how much of the observed variation is due to real change in the heliospheric ENA flux. Although the uncertainties are large, a lot of the variation seems to trend consistently over consecutive points. If it were solely statistical, we would expect more point-to-point fluctuation. Interestingly, the pattern in the north is similar to what is seen in Figure 23 of McComas et al. (2014) for the "nose/north pole" region, at least through 2013 , even though their sample includes a much greater portion of the sky than just a single $6^{\circ} \times 6^{\circ}$ polar pixel. On the other hand, because of the large overlap in the ESA passbands (see Figure 18, Funsten et al. 2009a), the flux rates observed in two adjacent ESA steps are not necessarily independent, and we expect to see some correlation between the signals in each. This is clearly not the case for ESAs 2 and 3, especially in the north. Thus, if the time variation in ESA 2 is real, it leads us to suspect that it must be occurring predominantly on the lowenergy side of the energy range, that is, below $710 \mathrm{eV}$.

\subsection{IBEX-Lo Polar Fluxes}

We turn to $I B E X$-Lo to investigate the low-energy ENA behavior further. In general, the IBEX-Lo instrument does not have sufficient sensitivity to investigate time variation in the ENA flux, as the count rates are too low to draw statistically meaningful conclusions. (Note this statement does not apply to the interstellar neutral signal, for which the statistics are excellent (see, e.g., Möbius et al. 2012; McComas et al. 2015). However, just as for $I B E X$-Hi, IBEX-Lo continuously observes the ecliptic poles, so this is the one place in the sky where lowenergy ENAs (0.20-1.0 keV) can be observed with reasonable statistics. Figure 4 shows north and south polar fluxes for Lo ESAs L5, L6, and L7, corresponding to central passband 

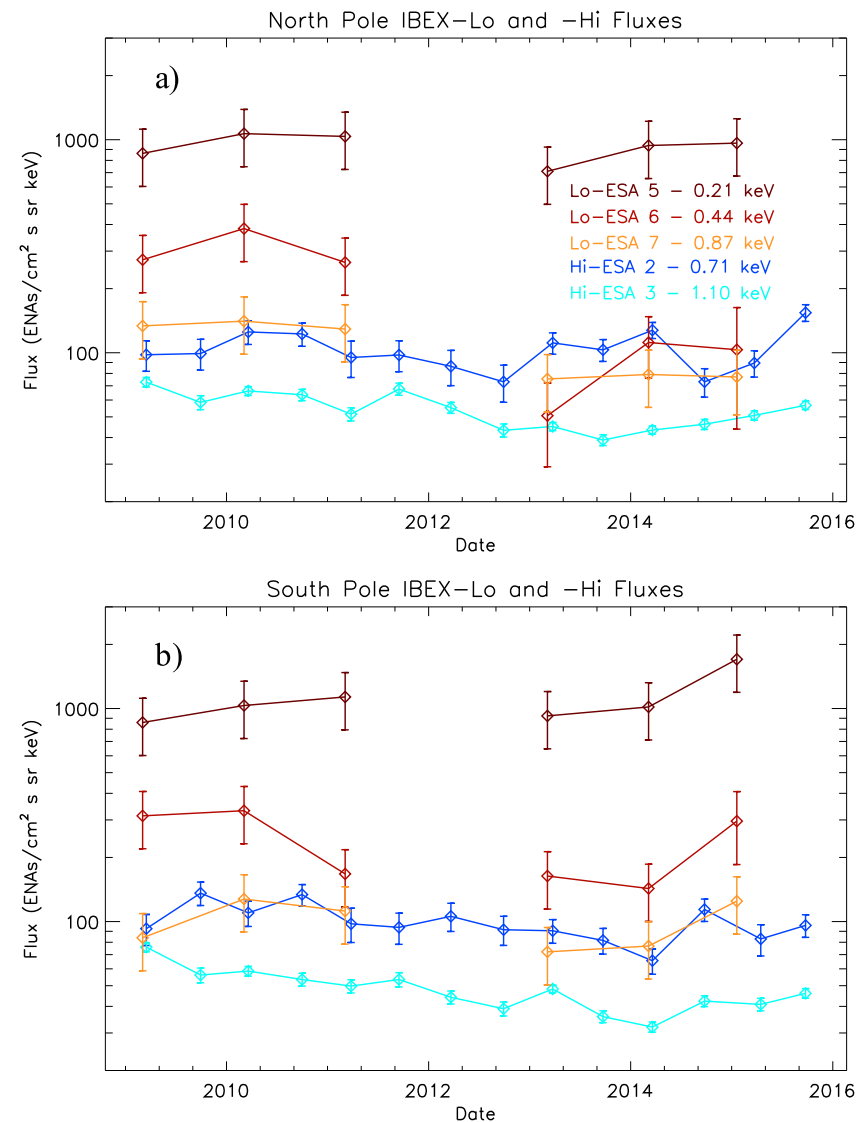

Figure 4. $I B E X$-Lo differential ENA energy fluxes at the (a) north and (b) south ecliptic poles for IBEX-Lo ESAs 5, 6 and 7. Each IBEX-Lo point represents roughly four months of data; error bars show the corresponding $1 \sigma$ statistical uncertainties. The six-month $I B E X$-Hi fluxes for ESAs 2 and 3 from Figure 2 are replotted for comparison. Note that the instrument calibration uncertainties for both $I B E X$-Hi and $I B E X$-Lo are $~ 30 \%$; thus, the data from a given sensor may be collectively shifted relative to the other sensor by $\pm 30 \%$ and still be consistent with calibration.

energies of $0.21,0.44$, and $0.87 \mathrm{keV}$, respectively. Note that Lo ESA L7 and Hi ESA $2(0.71 \mathrm{keV})$ are close in energy, and thus Lo ESA L7 can serve to independently validate Hi ESA 2.

Each IBEX-Lo point in Figure 4 is an average of four months of data, but the points are spaced a year apart because the Lo signal is overwhelmed by magnetospheric contamination during the latter two-thirds of the year, rendering the data nearly unusable (see, however, Fuselier et al. 2012). A data point for 2012 is not shown as there seems to be a high level of contamination of unclear origin for that entire year. The Lo data are corrected for survival probability, just as the Hi data are. Error bars reflect the statistical uncertainties of the counts and the uncertainties associated with the subtraction of backgrounds (see Galli et al. 2014 for a detailed description of the IBEX-Lo ENA analysis method). An absolute calibration uncertainty of $\pm 30 \%$, which is not included in the error bars, should also be applied. It should also be noted that, starting in 2013 , the IBEX-Lo PAC voltage was decreased from $16 \mathrm{kV}$ to $7 \mathrm{kV}$. This reduced the sensitivity of the sensor and required a change in the geometric factor used to calculate the fluxes.

In most cases, the variations in the Lo fluxes are just at the limit of statistics, making it difficult to conclude if any real heliospheric variation is present. It does appear, however, that a significant drop occurs for Lo ESAs 6 and 7 between 2011 and 2013 that persists thereafter, particularly in the north. At the
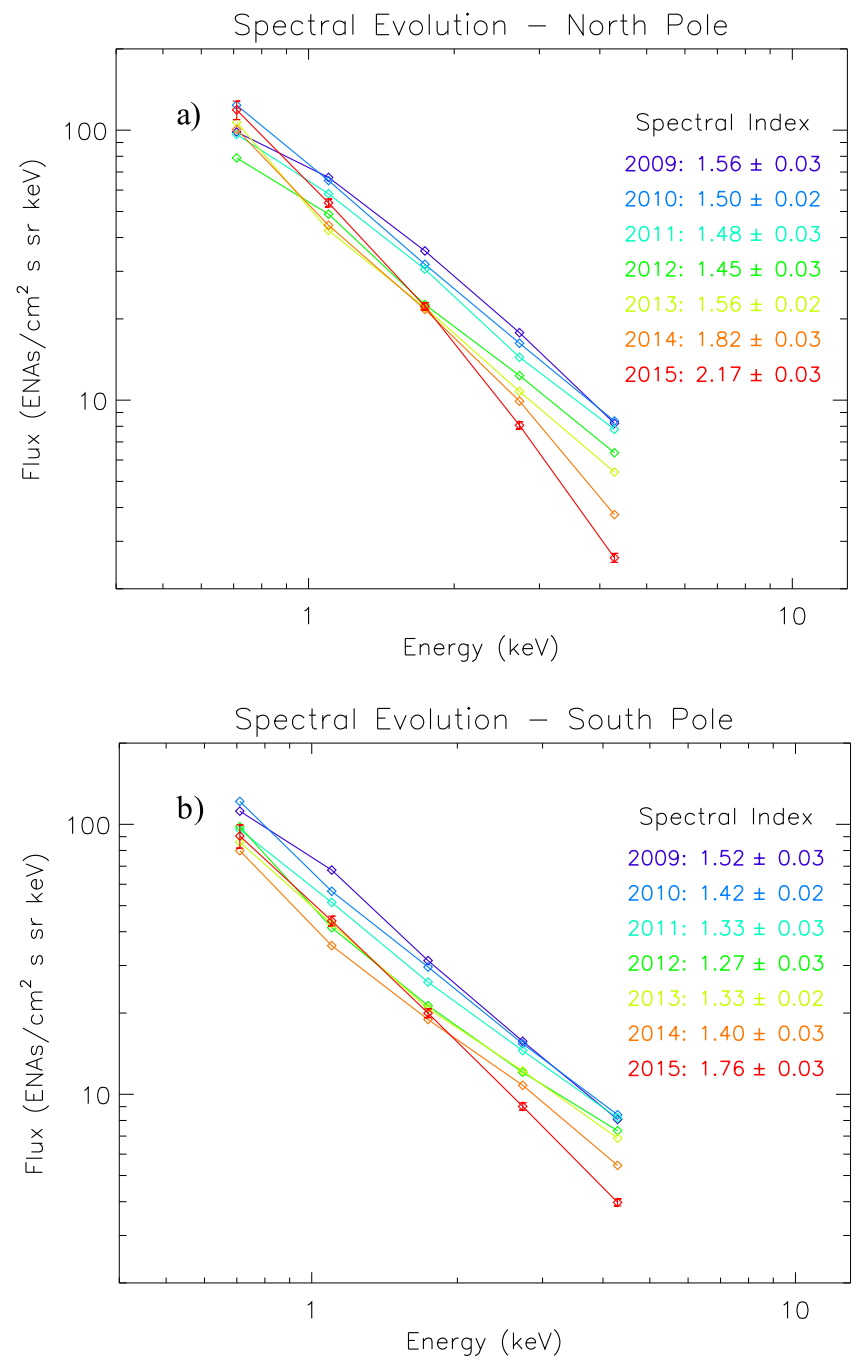

Figure 5. $I B E X$-Hi differential ENA flux vs. energy at the (a) north and (b) south ecliptic poles, each colored curve corresponding to a yearly average of the flux. The color progression from purple to red corresponds to a yearly time progression from 2009 to 2015 . Representative error bars showing the $1 \sigma$ uncertainty are shown for the latest spectrum. Also shown are the spectral indices $\gamma$ from power-law fits $\left(E^{-\gamma}\right)$ to the energy spectra and the uncertainties in the fits.

south pole, an upturn occurs in 2015 which, although perhaps real, is likely a reflection of increased background rates. Because of the very low count rates, we are dealing with Poisson rather than Gaussian statistics, and as the data get noisier, there are more positive outliers than negative, and hence even though the background is subtracted, the remaining count rate is higher because of the asymmetric nature of fluctuations for Poisson statistics.

For comparison to $I B E X$-Lo, we also show in Figure 4 the ENA fluxes measured by IBEX-Hi ESAs 2 and 3 . We see that the Lo ESA 7 points overlap the Hi ESA 2 data. Thus, although it is not possible to make conclusive statements about the time variation based on the IBEX-Lo data alone, they do support the case that the variations seen in Hi ESA 2 are most likely of heliospheric origin.

\subsection{Energy Spectra}

A complementary way to visualize the time variation is to examine the evolution of the ENA energy spectra over time. 
Figure 5 shows ENA spectra spaced at yearly intervals for the north and south poles. Each curve corresponds to consecutive 12-month averages of the flux. This further reduces the statistical uncertainty, as indicated by the (very small) statistical error bars on the 2015 spectra, and calls out the long-term trend.

To quantify the change in spectral shape, we fit each spectrum to a power law parameterized by a single spectral index $\left(E^{-\gamma}\right)$. We do not necessarily expect the ENA spectrum to follow a simple power law at these energies, and in fact several studies have been published characterizing the spectra with multiple spectral indices (e.g., Funsten et al. 2009b; Dayeh et al. 2011, 2012; Schwadron et al. 2011; Allegrini et al. 2012; Desai et al. 2015); rather, we are attempting to quantify the time evolution with a single parameter.

The best-fit spectral indices $\gamma$ are shown in Figure 5, along with the fit uncertainties. For all spectra, the uncertainties in $\gamma$ are no greater than \pm 0.03 , so statistically significant change is observed. The values of $\gamma$ indicate that the ENA fluxes from the two poles evolve somewhat differently. At the north pole, $\gamma$ remains essentially constant at 1.5 for the first five years. The flux decline is generally slow at first, but it then accelerates rapidly at all energies between 2011 and 2012. From 2014 on, the flux decline at high energies accelerates even more rapidly while the low-energy flux begins to recover, resulting in a spectral index that steepens to 2.1. At the south pole, $\gamma$ begins at 1.5 in 2009 but then shallows to 1.3 by 2011 as the lowenergy flux falls while the high-energy flux stays flat. The spectral index remains steady at $\sim 1.3$ through 2013 as the flux at all energies drops at roughly the same rate. In 2014 the spectral index begins to steepen, falling to 1.8 by 2015 , as the flux at low energies recovers while at high energies the decline accelerates. The overall picture we are left with is that both poles show the same basic evolution, but that the south lags the north by perhaps a year. One might conclude from this that the heliospheric boundary in the south is farther away than in the north, but as we will see in Section 4.4, this is instead a result of differences in the evolution between the northern and southern hemispheres of the solar atmosphere over the course of the solar cycle.

\section{ANALYSIS AND DISCUSSION}

We now explore the relationship between time variations in the polar ENA fluxes and the solar wind. R2012 discusses that solar wind variation can cause changes in the ENA flux through two mechanisms: first, variations in the SW dynamic pressure impinge on the TS, setting up pressure waves that propagate through the IHS at the local magnetosonic speed, affecting the flux of ENAs formed there; and second, an imprint of temporal structure in the pre-TS solar wind may still be present in the shocked plasma streaming through the IHS, which then carries over to the ENAs. We contend that both mechanisms are at work and have observable consequences.

\subsection{Pressure Wave Propagation}

The first, the action of the SW dynamic pressure at the TS, is reflected in the total ENA flux or, more precisely, in the calculated plasma pressure integrated over the LOS through the ENA formation region (see, e.g., Schwadron et al. 2011). This quantity is derived from integrating the ENA flux over energy:

$$
P_{\mathrm{s}} \cdot L=\frac{2 \pi m^{2}}{3 n_{H}} \int_{E_{\min }}^{E_{\max }} \frac{d E}{E} \frac{j_{\mathrm{ENA}}(E)}{\sigma(E)}(\mid v)^{3} .
$$

The limits of integration span the range of energies for which we have direct observations of the ENA flux $j_{\mathrm{ENA}}(E)$, where $E$ is the ENA energy in the Sun's rest frame, and $v$ is the corresponding velocity. Note that for ecliptic polar observations, the energy in the solar frame is the same as it is in the instrument frame, since the spacecraft's motion relative to the Sun is transverse to the poles. Thus, no transformation is required to translate the measured energy to the Sun's inertial frame. In Equation (1), $m$ is the proton mass, $n_{\mathrm{H}}$ is the interstellar neutral hydrogen density, and $\sigma(E)$ is the chargeexchange cross section (Lindsay \& Stebbings 2005).

The quantity $P_{\mathrm{s}}$ denotes the "stationary" pressure, or the pressure of the IHS plasma if it were at rest in the solar frame. (Strictly speaking, the only condition is that the plasma has no radial motion relative to the Sun (see R2012 and Schwadron et al. 2011).) The distance $L$ is the thickness of the primary ENA-forming region, interpreted here as the thickness of the IHS. The product $P_{\mathrm{s}} \cdot L$ is referred to as the "LOS-integrated pressure," and it is entirely determined from the observations.

We expect $P_{\mathrm{s}}$ to be correlated with the SW dynamic pressure for the following reason: by far, the majority of the energy in the outflowing supersonic SW is contained in the dynamic pressure $\left(\rho v^{2}\right)$ rather than in the internal pressure $(n k T)$. Based on the SW mass-loading model described by Schwadron et al. (2011, 2014), we find that at the TS, the dynamic pressure comprises $\sim 98 \%$ of the total pressure and is responsible for maintaining the inflation of the heliosphere. The remaining $2 \%$ is due primarily to the internal pressure of pickup ions traveling with the solar wind. Downstream of the TS, although the internal pressure dramatically increases and the dynamic pressure correspondingly decreases, the total pressure across the TS will remain in rough balance; thus, a change in the SW dynamic pressure will lead to a corresponding change in the pressure within the IHS and, via Equation (1), the ENA flux. The action of this mechanism can be seen in time-varying MHD models of the outer heliosphere (see in particular Washimi et al. 2011, Figures 5 and 6).

There are three important points to make here. First, the IHS pressure is derived from the ENA flux integrated over all energies; thus, although we expect the total ENA flux to be correlated with the SW dynamic pressure, it is not necessarily required that the ENA flux at a particular energy be correlated. Second, ENA flux variations due to this mechanism will be associated with dynamic pressure changes incident on the TS at points within a region $\sim 15^{\circ}$ wide, noseward of the direction in which the ENAs are viewed by IBEX. This is because pressure disturbances propagate predominantly radially outward through the IHS. There will be some convection of disturbances tailward due to the plasma flow in the IHS, but at the poles, the transverse component of the plasma flow is expected to be $\lesssim 100 \mathrm{~km} \mathrm{~s}^{-1}$, which is small compared to the magnetosonic speed of $\sim 450 \mathrm{~km} \mathrm{~s}^{-1}$. Third, as pointed out by Schwadron et al. (2011) and R2012, the nonstationary component of the LOS-integrated pressure scales linearly with the stationary component; thus, for the purpose of correlating changes in the IHS with the 1 au SW dynamic pressure, it is sufficient to work with $P_{\mathrm{s}} \cdot L$. 


\subsection{Propagation of Variations along Streamlines}

The ENAs detected by $I B E X$ originate from a population of pickup ions and SW protons whose outward flow has been slowed and heated at the TS and then diverted along the flanks and over the poles of the heliosphere toward the heliotail. It is reasonable to expect that the ENA flux should retain some memory of the physical state of the outbound solar wind from which it originated. Consider the flux of ENAs incident on IBEX from a particular look direction. The line of sight in this direction intercepts a collection of IHS streamlines that can be traced to solar wind originally propagating outward from the Sun along radials arranged between the pressure maximum in the heliosheath (located $20^{\circ}$ south of the nose; McComas \& Schwadron 2014) and the look direction of IBEX. The ENA flux should correlate in some manner with the properties of the outbound solar wind averaged over these radial directions. In the case of observations of the ecliptic poles, we are then in principle considering the SW flux averaged over all latitudes from the heliosheath pressure maximum to the pole and over a narrow wedge of longitudes centered on the upwind direction.

Determining exactly how the physical state of the progenitor solar wind will be imprinted onto the ENA flux is not straightforward. Three factors act to mitigate the transfer of information, particularly in the case of ENAs observed at the poles.

The first factor is the spread in proton travel times along streamlines that enter the heliosheath at different latitudes and then continue through the IHS to their respective points of intersection with the LOS to IBEX. Based on a survey of outer heliospheric models (e.g., Pogorelov et al. 2007, 2013; Izmodenov et al. 2009; Opher et al. 2009, 2015), a proton outbound along the heliographic equator will travel roughly an additional 200 au through the IHS to the pole as compared to a proton traveling directly poleward. For a flow speed in the IHS of $\sim 150 \mathrm{~km} \mathrm{~s}^{-1}$ (based on Voyager 2 observations; Burlaga et al. 2009), this corresponds to a travel time difference of $\sim 6.5$ years, or more than half a solar cycle. Thus even if the individual streamlines retain memory of their preshock solar wind conditions, ENAs observed along a polar-directed LOS will reflect SW variability averaged over half a solar cycle. Some heliospheric models predict even longer path lengths along streamlines from the nose to the pole (e.g., Florinski et al. 2005), in which case the averaging timescale may be considerably longer.

The second mitigating factor is the lifetime of protons in the heliosheath. The proton population to which IBEX-Hi is sensitive has energies ranging from 0.5 to $6 \mathrm{keV}$. For a neutral density of $n_{\mathrm{H}}=0.1 \mathrm{~cm}^{-3}$, the proton lifetime (the $1 / e$ time) ranges from $\sim 2.8$ years (at $6 \mathrm{keV}$ ) to $\sim 5$ years (at $0.5 \mathrm{keV}$ ). Thus only a small fraction of low-latitude SW protons will survive a journey to the heliographic poles, ranging from $\sim 15 \%$ of protons at $6 \mathrm{keV}$ to $\sim 30 \%$ of protons at $0.5 \mathrm{keV}$. Hence, the vast majority of polar ENAs will originate from SW protons initially outbound at relatively high latitudes. This limits the impact of the first factor: instead of the ENA flux being averaged over half a solar cycle or more, ENAs will originate from a population of mostly high-latitude solar wind, averaged over $2-4$ years.

The third factor is the tendency for pressure variations in the IHS to be smoothed because the magnetosonic speed is faster than the plasma flow speed by a factor of about three. Thus pressure structures in the SW flow that have streamed across the TS will tend to be washed out before they can show up in the ENA flux.

Exactly how the interplay of these factors affects the ENA flux can only be determined by detailed time-dependent numerical modeling of a structured, time-varying solar wind; nevertheless, it is evident that the combined action of these three effects limits the amount of pre-TS SW structure that can imprint on the polar ENA flux observed by IBEX, particularly for the higher-energy ESA steps. We thus conclude that the variation observed in the ENA flux will be due primarily to pressure waves in the heliosheath driven by the SW dynamic pressure exerted on the TS, and due secondarily to structures flowing in the IHS plasma that have crossed the TS within a few tens of degrees noseward of the ENA origination site. We will next show that evidence for both sources of ENA variability is present.

\subsection{Evidence for the Effect of Pressure Waves on the ENA Flux}

As was done in R2012, we can test our assertion that variations in the polar ENA flux are driven by variations in the SW dynamic pressure by correlating the SW dynamic pressure measured at 1 au, $P_{1 \text { au}}$, with the LOS-integrated pressure, $P_{\mathrm{s}} \cdot L$ calculated from Equation (1). We now have seven years of ENA data to use in the correlation, as opposed to only two years in the original study, so the test should be correspondingly more rigorous.

\subsubsection{Trace-back Time}

In R2012, the concept of "trace-back time" was introduced in order to determine the $1 \mathrm{au} \mathrm{SW}$ observations that are appropriate to compare to IBEX ENA observations. The traceback time is the time between when ENAs of a given energy are observed by $I B E X$ and the prior observation time of the outgoing 1 au solar wind that eventually influences the environment where the ENAs are formed. We estimate the trace-back time as

$$
\left\langle t_{\mathrm{tb}}(E)\right\rangle=\frac{d_{\mathrm{TS}}}{v_{\mathrm{sw}}}+\frac{l_{\mathrm{IHS}} / 2}{v_{\mathrm{ms}}}+\frac{d_{\mathrm{TS}}+l_{\mathrm{IHS}} / 2}{v_{\mathrm{ENA}}(E)}
$$

where $\left\langle t_{\mathrm{tb}}(E)\right\rangle$ is the average trace-back time for ENAs of energy $E$ originating in the IHS, $d_{\mathrm{TS}}$ is the distance to the TS, $l_{\mathrm{IHS}}$ is the thickness of the IHS, $v_{\mathrm{sw}}$ is the SW proton speed, $v_{\mathrm{ms}}$ is the average magnetosonic speed in the IHS, and $v_{\mathrm{ENA}}(E)$ is the speed of an ENA of energy $E$ observed at $I B E X$. Note that $l_{\mathrm{IHS}}$ and the parameter $L$ introduced in Equation (1) describe the same quantity; however, we use the notation $l_{\mathrm{IHS}}$ to make it clear we are assuming the ENA source is primarily the IHS.

The first term on the right-hand side of Equation (2) describes the time it takes for a solar wind parcel traveling outbound from the Sun to reach the TS. The second term is the time it takes a pressure pulse generated at the TS traveling at the magnetosonic speed to propagate to the midpoint of the ENA-forming region. The last term describes the time it takes ENAs formed at this point to travel back to IBEX. Of course, ENAs will be generated along the whole distance from $d_{\mathrm{TS}}$ to $d_{\mathrm{TS}}+l_{\mathrm{IHS}}$, and so in reality there is a range of travel times. Thus, in our parameterization, we are making the simplifying assumption that all ENAs originate from the midpoint of this region. Note also that ENAs at different energies have different trace-back times. This means that to carry out the pressure correlation, the energy integral in Equation (1) must be 
performed over ENA flux measurements observed at different times, as determined by the different trace-back times, to arrive at a value of $P_{\mathrm{s}} \cdot L$ appropriate for comparison to a specific measurement of $P_{1 \text { au}}$.

The values adopted for the distances in Equation (2) are now considered. The distance to the midpoint of the ENA emission region, $d_{\mathrm{ENA}}=d_{\mathrm{TS}}+l_{\mathrm{IHS}} / 2$, is calculated by the method described in Section 4.3.2. Since this distance depends on two unknowns ( $d_{\mathrm{TS}}$ and $\left.l_{\mathrm{IHS}}\right)$, this procedure results in an infinite set of $\left(d_{\mathrm{TS}}, l_{\mathrm{IHS}}\right)$ combinations. Thus, it is necessary to separately constrain at least one of these to arrive at a unique $\left(d_{\mathrm{TS}}, l_{\mathrm{IHS}}\right)$ pair. The values for $d_{\mathrm{TS}}$ can be reasonably estimated by considering the Voyager 1 and 2 TS crossing distances of 94 au and 84 au, respectively (Burlaga et al. 2005; Stone et al. 2008), and using the results of heliospheric models to scale these distances to the poles. Based on a survey of asymmetric heliosphere models (e.g., Pogorelov et al. 2007, 2013; Opher et al. 2009, 2015; Izmodenov et al. 2009), we assign a value of $d_{\mathrm{TS}}=130 \mathrm{au}$ at the north pole and $d_{\mathrm{TS}}=110 \mathrm{au}$ at the south pole. Then, the values for $l_{\mathrm{IHS}}$ are varied until the best correlation between $P_{1 \text { au }}$ and $P_{\mathrm{s}} \cdot L$ is found. As will be described in Section 4.3.2, this method leads to best-fit IHS thickness values of $l_{\mathrm{IHS}}=210 \mathrm{au}$ in the north and $l_{\mathrm{IHS}}=160 \mathrm{au}$ in the south.

Turning to the speeds used in Equation (2), at the poles we expect, at least for most of the solar cycle, the speed of the outgoing solar wind to be $\sim 755 \mathrm{~km} \mathrm{~s}^{-1}$ in the inner heliosphere $(<5 \mathrm{au}$ ), based on measurements from Ulysses (McComas et al. 2000; Ebert et al. 2009). As the solar wind travels outward, it steadily slows, due to mass loading by pickup ions. We use the one-dimensional mass-loading model of Schwadron et al. $(2011,2014)$ to track the proton speed out to the TS, finding that it falls linearly with distance to about $610 \mathrm{~km} \mathrm{~s}^{-1}$ by $120 \mathrm{au}$, the average of our estimates of the distances to the TS at the poles. Averaging the speed from 1 au out to $120 \mathrm{au}$, we arrive at a value of $v_{\mathrm{sw}}=690 \mathrm{~km} \mathrm{~s}^{-1}$ for use in Equation (2). For the magnetosonic speed in the IHS, we use the value of $v_{\mathrm{ms}}=430 \mathrm{~km} \mathrm{~s}^{-1}$ derived in R2012. Using these values for $d_{\mathrm{TS}}, l_{\mathrm{IHS}}, v_{\mathrm{sw}}$ and $v_{\mathrm{ms}}$, the ENA trace-back times range from 4.9 to 3.1 years in the north and 4.0 to 2.5 years in the south, for ESAs 2 through 6, respectively.

\subsubsection{Correlation of 1 au Dynamic Pressure with ENA-derived Plasma Pressure}

In practice, since ENAs are measured in discrete energy passbands, the integral in Equation (1) must be calculated in a piece-wise manner. We assume that $j_{\mathrm{ENA}}\left(E_{i}\right)$ is a measurement of the flux precisely at the central energy $E_{i}$ of the $i$ th passband, and that the flux between adjacent central energies follows a power law. (The assumption that $j_{\text {ENA }}$ should be fixed at the central energy will lead to a small overestimation of the flux spectrum, but since we are interested here primarily in variations, this does not significantly impact our analysis.) We then calculate a spectral index $\gamma_{i}$ for the flux between $E_{i}$ and $E_{i+1}$ as

$$
\gamma_{i}=-\frac{\log \left(j_{\mathrm{ENA}}\left(E_{i+1}\right)\right)-\log \left(j_{\mathrm{ENA}}\left(E_{i}\right)\right)}{\log \left(E_{i+1}\right)-\log \left(E_{i}\right)}
$$

Then, the LOS-integrated pressure is given by the sum of a set of LOS-integrated partial pressures:

$$
\Delta\left(P_{\mathrm{s}} \cdot L\right)_{i} \cong \frac{4 \pi \sqrt{2 m}}{3 n_{H}} \int_{E_{i}}^{E_{i+1}} \frac{\sqrt{E}}{\sigma(E)} j_{\mathrm{ENA}}\left(E_{i}\right)\left(\frac{E}{E_{i}}\right)^{-\gamma_{i}} d E .
$$

Because the arrival time for ENAs from a common point in the heliosheath depends on the ENA energy, the total LOSintegrated plasma pressure $P_{\mathrm{s}} \cdot L$ to associate with a particular 1 au dynamic pressure measurement $P_{1 \text { au }}$ will be the sum of partial pressures computed from ENA fluxes having different trace-back times but a common choice of the distances $d_{\mathrm{TS}}$ and $l$. Thus we shift the $\Delta\left(P_{\mathrm{S}} \cdot L\right)_{i}$ time series for each ENA energy separately as dictated by the trace-back time for that energy, and then we sum the partial pressures for the instances where the trace-back times for all five energy steps are the same. This is illustrated in Figure 6, which plots the partial LOS pressures versus time, along with the 1 au SW dynamic pressure, $P_{1 \text { au }}$. Because the SW dynamic pressure is regarded as an SW invariant with heliolatitude (McComas et al. 2008), we use the dynamic pressure calculated from the ecliptic OMNI-2 archive (King \& Papitashvili 2005) as a proxy for $P_{1 \text { au }}$ in the polar regions (see also the discussion in Sokół et al. 2013 and Sokół et al. 2015). The upper plot is for the north pole, and the lower is for the south pole. The color-coded points in the lower portion of each plot are the LOS-integrated partial pressures $\Delta\left(P_{\mathrm{s}} \cdot L\right)_{i}$, each time-shifted by trace-back times computed from the best-fit values for $d_{\mathrm{TS}}$ and $l$ determined below.

For each pole, one can see from Figure 6 that there is a time range where all five partial pressures overlap. For these periods, the total pressure can be calculated, shown as the black line in each plot. Note that the total LOS pressure is well correlated with the SW dynamic pressure (red line), even though the individual partial pressures are not. This demonstrates the point made in Section 4.1 that the ENA flux at a specific energy need not track $P_{1 \text { au }}$ for there still to be a good correlation between the total LOS pressure and $P_{1 \text { au }}$. What determines the ENA flux at a specific energy will be discussed in Section 4.4.

The procedure for determining the set of trace-back times that leads to the best correlation between $P_{\mathrm{s}} \cdot L$ and $P_{1 \text { au }}$ is illustrated in Figure 7, which shows scatter plots of measurements of $P_{\mathrm{s}} \cdot L$ versus $P_{1}$ au for the north and south poles. The overlap periods are the same as in Figure 6. In each plot, in addition to a solid line representing a linear fit to the points, we have drawn a dashed line that runs from the midpoint of the linear fit to the origin $(0,0)$. The individual partial pressures $\Delta\left(P_{\mathrm{s}} \cdot L\right)_{i}$ that comprise $P_{\mathrm{s}} \cdot L$ were time-shifted by varying $l_{\mathrm{IHS}}$ to minimize the difference between the slopes of the linear fit line and the zero-intercept line.

If the linear fit line were to lie directly along the zerointercept line, this would mean a one-to-one correspondence exists between the 1 au dynamic pressure and the ENA-derived heliosheath pressure. From this, one would conclude that the heliosheath pressure is rigidly coupled to the SW dynamic pressure. The fact that the linear fit lines have shallower slopes than the zero-intercept lines may be due to some combination of the following: (1) Because the observed ENAs do not originate at a single point but rather along a poleward path through the IHS having a thickness that is presumably $\gtrsim 100 \mathrm{au}$, the ENAs observed by IBEX-Hi have a large range of origination times, which has the effect of "smoothing" the pressure response. (2) Because some of the observed ENA flux 

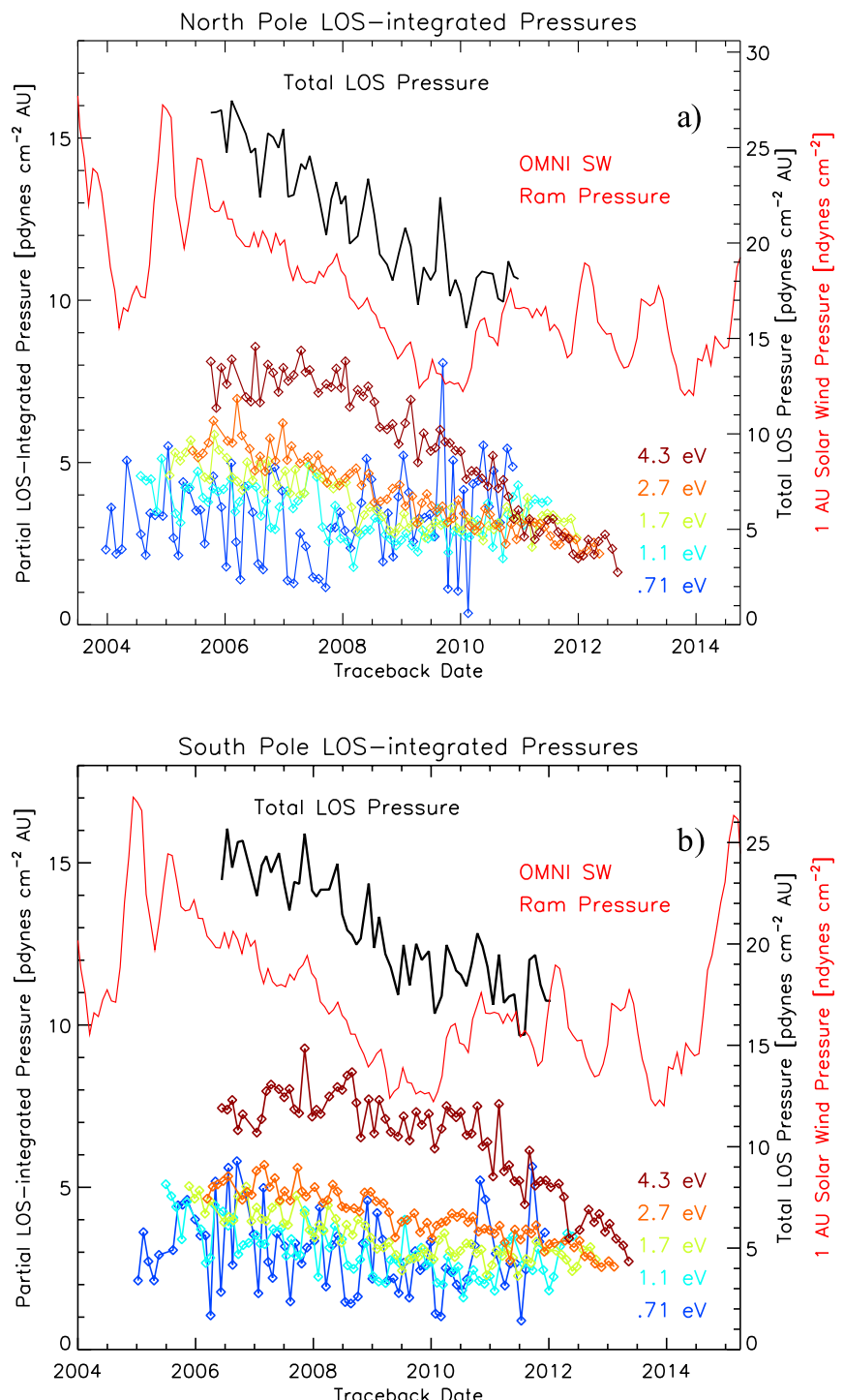

Figure 6. Partial and total LOS-integrated plasma pressures in the IHS vs. trace-back time (Equation (2)), at the (a) north and (b) south ecliptic poles. The partial LOS-integrated pressures are derived using Equation (4) from the ENA fluxes shown in Figure 2, using the same color coding. The total LOSintegrated pressure (black curve) is calculated from the partial pressures (Equation (1)) for the range when the trace-back times for all five ESAs overlap. Also shown is the 1 au SW dynamic (ram) pressure calculated from the OMNI data set (red line) plotted for the actual time of observation. The trace-back times are based on the best correlation between the total LOSintegrated plasma pressure and the 1 au SW dynamic pressure.

may originate beyond the region directly influenced by the solar wind, such as the outer heliosheath, there may be a component of $P_{\mathrm{S}} \cdot L$ that is uncorrelated with the SW pressure. (3) Discussed in more detail at the end of this section, the integral in Equation (1) does not cover the full range of energies that contribute significantly to the heliosheath plasma pressure, so a one-to-one correspondence is not necessarily expected. Rather, some of the variation in the ENA flux may be due to variations along streamlines, as described in Section 4.2.

For the moment, we assume that the observed variation in $P_{\mathrm{S}} \cdot L$ presented here is driven solely by the $\mathrm{SW}$ dynamic pressure. As discussed in Section 4.3.1, there is no one unique combination of $d_{\mathrm{TS}}$ and $l_{\mathrm{IHS}}$ that minimizes the slope difference. In Section 5 we will discuss separately constraining $d_{\mathrm{TS}}$ and $l_{\mathrm{IHS}}$, but for this work, we adopt the values for the
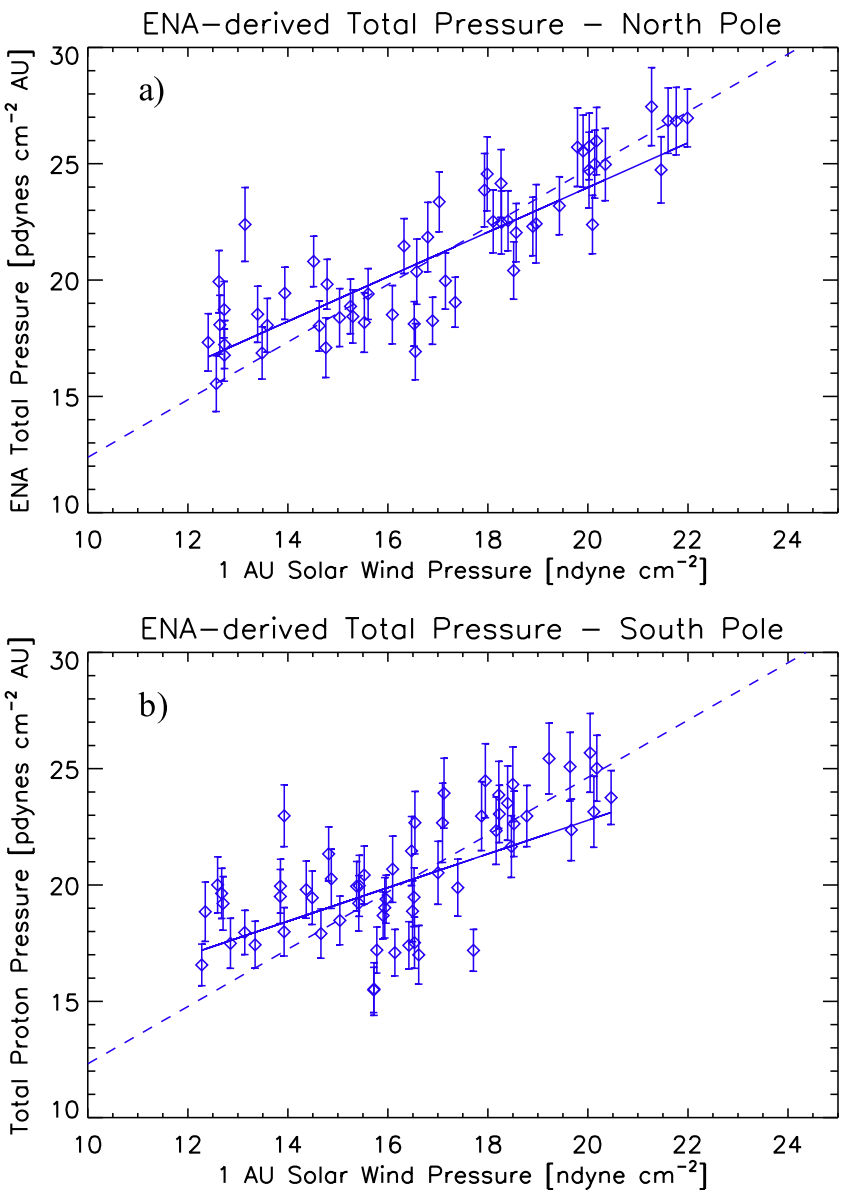

Figure 7. Total LOS-integrated partial pressure in the IHS derived from ENA flux vs. 1 au SW dynamic pressure for the (a) north and (b) south ecliptic poles. The solid line is a linear fit to the data points; the dashed line is drawn from the midpoint of the linear fit to the origin and indicates a one-to-one correspondence between changes in the IHS total LOS pressure and the $1 \mathrm{au}$ dynamic pressure.

inferred poleward TS distances based on Voyager TS crossing distances as mentioned above. For the adopted value of $d_{\mathrm{TS}}=130 \mathrm{au}$ in the north, the slope-minimization method results in an IHS thickness of $l_{\mathrm{IHS}} \sim 210 \mathrm{au}$. For the south, the adopted value of $d_{\mathrm{TS}}=110$ au leads to an IHS thickness of $l_{\mathrm{IHS}} \sim 160 \mathrm{au}$. Another way to report the dimensions of the heliosphere is to calculate the distance to the center of the ENA emission region, $d_{\mathrm{ENA}}=d_{\mathrm{TS}}+l_{\mathrm{IHS}} / 2$. Although the method used here does not separately constrain $d_{\mathrm{TS}}$ and $l_{\mathrm{IHS}}$, it turns out that $d_{\text {ENA }}$ varies only weakly with the choice of the best-fit $\left(d_{\mathrm{TS}}, l_{\mathrm{IHS}}\right)$ combination. The present analysis predicts that the distances to the center of the ENA source region are $d_{\mathrm{ENA}}=220 \mathrm{au}$ in the direction of the north ecliptic pole and $d_{\mathrm{ENA}}=190 \mathrm{au}$ in the direction of the south ecliptic pole.

The derived north pole IHS thickness value of $l_{\mathrm{IHS}} \sim 210 \mathrm{au}$ is within the range predicted by asymmetric heliosphere models, although there is considerable variation, from $100 \mathrm{au}$ (Pogorelov et al. 2013) to 240 au (Izmodenov et al. 2009). The derived IHS thickness in the south is less than in the north pole, presumably due to compression of the heliosheath along the southern leading edge by the interstellar magnetic field. Still, the south pole value of $160 \mathrm{au}$ is considerably larger than model predictions, most of which give a south pole IHS thickness of around 80-110 au. A notable exception is the magnetized polar jet model of Opher et al. (2015), who 
calculate a south pole IHS thickness of $175 \mathrm{au}$, which is consistent with our finding.

Further support for a thick heliosheath in the poleward directions comes from Galli et al. (2016), who estimate the dimensions of the IHS using IBEX-Lo data along with theoretical estimates of the IHS total pressure, which include both the stationary and dynamic pressure components (Schwadron et al. 2011, 2014). They average together the ENA flux in four macropixels, two in the downwind direction and two at the poles, to come up with an average "downwind" IHS thickness of $220 \pm 110 \mathrm{au}$. Although the heliosheath in the downwind direction is expected to be thicker than in the poleward direction, we point out that the fluxes at all four of their macropixels agree. What is different is the modeled total pressure, which is higher at the poles than downwind, leading to an IHS that is about $30 \%$ thinner at the poles than their $220 \mathrm{au}$ value. However, Galli et al. (2016) base their poleward pressure on the flow speed observed by Voyager 2 in the heliosheath, $140 \mathrm{~km} \mathrm{~s}^{-1}$, which originated as a slow SW $\left(\sim 400 \mathrm{~km} \mathrm{~s}^{-1}\right)$ prior to the TS (Burlaga et al. 2009). We argue that this is not appropriate for the poles over most of the solar cycle where the heliosheath flow speed should be considerably higher. As was done in R2012, if we assume a blend of flow speeds along the LOS at the poles ranging from $225 \mathrm{~km} \mathrm{~s}^{-1}$ (consistent with IHS flow from fast SW) to $140 \mathrm{~km} \mathrm{~s}^{-1}$, we derive a $\sim 200$ au IHS thickness at the poles from the Galli et al. (2016) fluxes.

We remind the reader that Voyager 1 observations suggest that the spacecraft passed through the heliopause at a distance of $122 \mathrm{au}$ and a heliolatitude of $\sim 35^{\circ} \mathrm{N}$, indicating a heliosheath thickness of just 28 au (Stone et al. 2013). This is well below the predictions of models, which typically derive IHS thicknesses $\gtrsim 40$ au in the Voyager 1 direction. On the other hand, Krimigis et al. (2011) used a combination of Voyager 1 and Cassini/INCA observations to empirically predict a thickness of $L_{V 1}=27_{-11}^{+26}$ in the IHS in the Voyager 1 direction. This calculation was further refined by Roelof et al. (2012) to include the Compton-Getting effect, arriving at an IHS thickness of $L_{V 1}=31_{-18}^{+31}$ au, which is in agreement with the Voyager 1 heliopause crossing observations. This method was then applied in the Voyager 2 direction by Roelof et al. (2012), predicting an IHS thickness of $L_{V 2}=71_{-20}^{+40}$ au. This is considerably thicker than the Voyager 1 IHS thickness, which is inconsistent with a heliosheath compressed in the southern hemisphere, as suggested by most asymmetric heliosphere models. (At the time of this writing, Voyager 2 has yet to reach the heliopause, so the prediction in the Voyager 2 direction is as yet unverified.)

We close this section by noting that if in fact the heliosheath in the polar directions is smaller than our determination, this is not necessarily in conflict with our analysis method. Recall that deriving the heliosheath thickness via the pressure correlation method requires that the integral in Equation (1) spans all energies that contribute significantly to the time-varying IHS pressure. For this study, we are limited to the energy range 0.5-6.0 keV; however, Cassini/INCA measurements of heliospheric ENAs show that a significant pressure component is present in the 5-13 keV range (Krimigis et al. 2009; Dialynas et al. 2015). There is also a significant contribution from energies below $0.5 \mathrm{keV}$, as indicated by Galli et al. (2016). Thus, if there is a different shape to the temporal variation in the flux from these portions of the energy spectrum, a pressure correlation analysis could possibly lead to dimensions that are consistent with a smaller heliosphere. Such a study is possible by combining the INCA and IBEX-Hi data sets; in fact, INCA heliospheric ENA observations do show time variations that may be correlated with the solar cycle (Dialynas et al. 2013). However, the IBEX-Lo sensor does not have the sensitivity necessary to carry out a time-resolved pressure correlation study with meaningful statistics. Thus, although perhaps a third of the total pressure will still be missing, an IBEX-Hi/INCA study could give us a more complete picture of heliosheath pressure variations over time.

\subsection{Evidence of Propagation of Solar Wind Structure along Streamlines}

Evidence for the presence of a solar wind structure that has survived passage across the TS and then continued to propagate along streamlines to the poles is not necessarily straightforward to find. As discussed in Section 4.2, the time resolution for variations will be of order 2-4 years. Thus the signal from SW transient structures, such as stream interfaces or coronal mass ejections, will be washed out. This means the only "structure" expected to be resolvable at the poles is changes in the SW energy distribution due to the solar cycle. Indeed, the fact that the ENA flux time series at different energies are not in phase even when the differing trace-back times are taken into account (see Figure 6) is in fact evidence of the changing SW energy distribution over the solar cycle.

In Section 4.2, we made the case that the polar ENAs will only be influenced by the SW within a few tens of degrees of the poles. Thus only the properties of the high-latitude SW should be reflected in the energy distribution of these ENAs.

The energy range of $I B E X-\mathrm{Hi}, 0.5-6 \mathrm{keV}$, is well above the bulk energy of the protons in the IHS. Even the protons from the originally $750 \mathrm{~km} \mathrm{~s}^{-1}$ high-speed polar wind observed by Ulysses, which has an energy of $\sim 3 \mathrm{keV}$ in the inner heliosphere, slow to $\sim 220 \mathrm{~km} \mathrm{~s}^{-1}$ after passage across the TS, which corresponds to an energy of only $250 \mathrm{eV}$. Thus, the source of ENAs observed by $I B E X-\mathrm{Hi}$ is predominantly pickup ions, but the energy of these ions will be linked to the energy of the pre-TS solar wind in which they were picked up.

Over the course of the solar cycle, one would expect the slope of the high-latitude pickup ion distribution to become shallower toward solar minimum and then steepen toward solar maximum, as the average speed of the solar wind at high latitudes oscillates between $\sim 750$ and $\sim 450 \mathrm{~km} \mathrm{~s}^{-1}$. This oscillation is due to the opening and closing of the polar coronal hole $(\mathrm{PCH})$, the source of the very high speed solar wind observed by Ulysses at high latitudes (McComas et al. 2000; Ebert et al. 2009).

This implies there should be a relationship between the energy spectra of the polar ENA flux and the size of the PCHs. To verify this, we compared the observations of the areas of the polar coronal holes to the ENA flux for the $4.2 \mathrm{keV}$ channel of IBEX-Hi over time. The PCH areas for both the north and south poles of the Sun have been determined from EUV images of the solar disk from a combination of Extreme-ultraviolet Imaging Telescope (EIT) images from the Solar and Heliospheric Observatory and Atmospheric Imager Assembly images from the Solar Dynamic Observatory (Karna et al. 2014).

Figure 8 shows the results of this comparison. The ENA flux and the polar coronal hole areas show an impressive degree of 

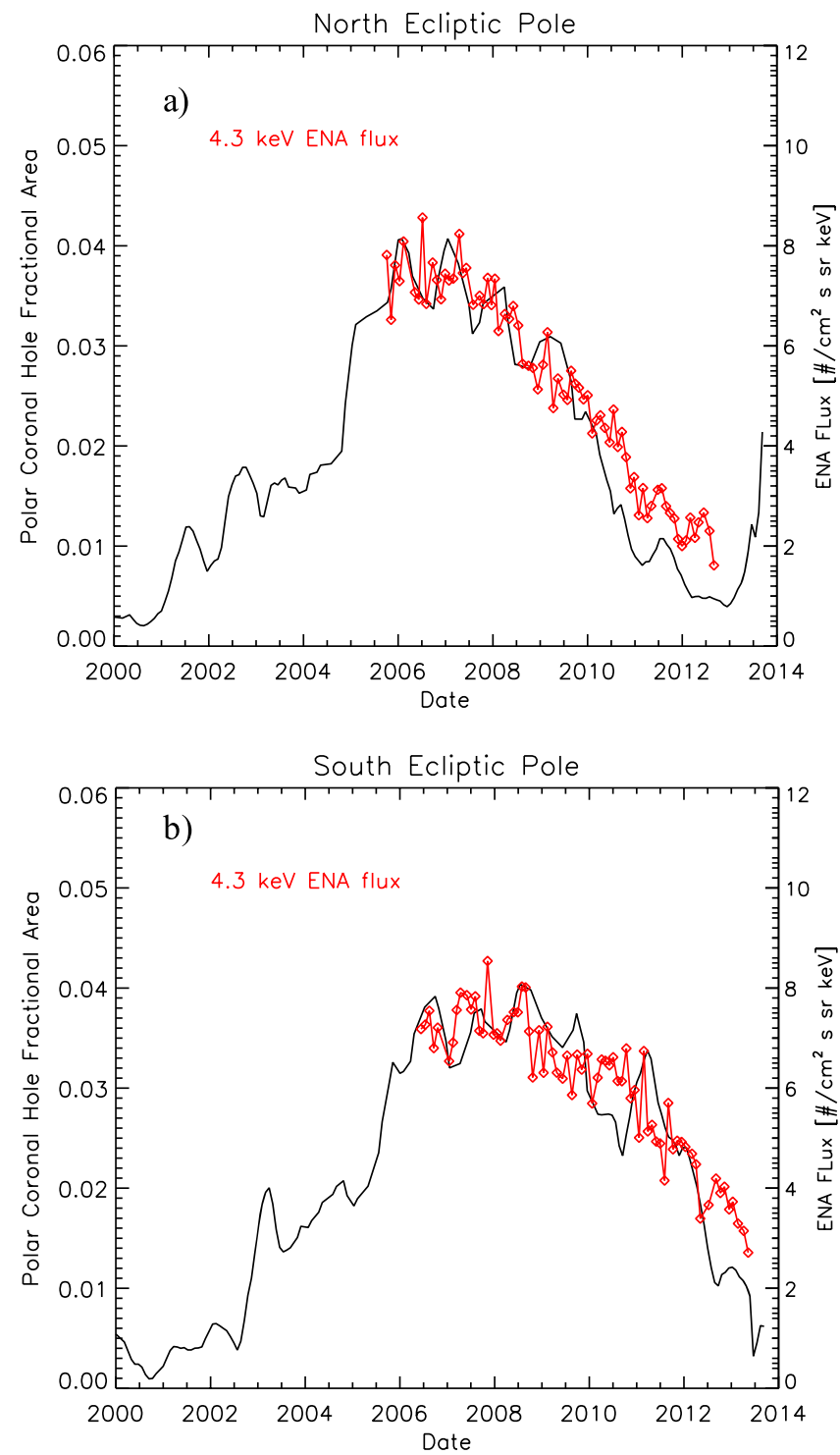

Figure 8. Comparison of the ENA flux (red) measured by the $I B E X-\mathrm{Hi} 4.3 \mathrm{keV}$ energy channel (ESA 6) with the fractional area (black) of the polar coronal hole for the (a) north and (b) south ecliptic poles. The PCH area data are based on the analysis method of Karna et al. (2014). The PCH data have been smoothed with a nine Carrington Rotation running average. The ENA data have been time-shifted by the same amount as shown in Figure 6. They have not been time-shifted in any way to match the PCH area data.

correlation for the period between 2006 and 2014, corresponding to the declining phase of SC23 and the ascending phase of SC24. We emphasize that the north and south pole ENA time series have the same time shifts as in Figure 6; they have not been shifted in any way to accommodate the PCH data. Note that the timing of the $\mathrm{PCH}$ growth between the poles is slightly different. By 2013, the area of the north PCH has begun to increase again, whereas at the south pole, there is no evidence of recovery. We see that the difference in trace-back times between the north and south ENA fluxes matches the difference in the timing of the $\mathrm{PCH}$ growth.

To further demonstrate the link between PCH area and ENA flux, Figure 9 shows a reconstruction of the SW speed versus latitude between 2000 and the end of 2013. These velocity evolution maps are based on interplanetary scintillation (IPS) data collected by the Institute for Space-Earth Environmental
Research (ISEE, formerly STEL) at Nagoya University, Japan (Tokumaru et al. 2012), and are routinely used by the IBEX team as input into the ENA survival probability model (McComas et al. 2012, 2014; Sokół et al. 2013, 2015). Here, we present the SW speed reconstruction to compare it to the $\mathrm{PCH}$ area. By comparison with Figure 8, we see that the latitudinal extent of the very fast solar wind $\left(v_{\mathrm{p}}>700 \mathrm{~km} \mathrm{~s}^{-1}\right)$ associated with $\mathrm{PCH}$ flow closely tracks the $\mathrm{PCH}$ areas. The evolution of the latitude structure of the fast SW also follows the phase offset of the PCH area between hemispheres.

Also clear from Figure 9, at low latitudes the SW speed distribution shows little variation over the course of the solar cycle. Thus, since the energy distribution of the ENAs observed at low latitudes should depend only on the lowlatitude SW, we expect the time variation of the ENA flux observed at low latitudes to be nearly in phase at all energies. To check this, we have averaged together the ENA flux in each sky map for all latitudes within $\pm 25^{\circ}$ of the ecliptic and longitudes within $\pm 90^{\circ}$ of the heliospheric nose, and then we plot the fluxes at different energies as a function of time (Figure 10). We limit the longitude range of the sample to the noseward hemisphere to ensure sufficient time resolution. In the tailward hemisphere, the path length through the IHS becomes increasingly longer such that the range of ENA travel times approaches and surpasses a solar cycle. The data are not corrected for the motion of the Earth in the Sun frame; thus we only use data from maps with the same viewing configuration (in this case, the odd-numbered sky maps). As the IBEX Ribbon is still of unexplained origin, we have been careful not to include pixels that contain the Ribbon in the average. This was done by using a mask based on the Ribbon separation scheme described in Schwadron et al. (2014).

Figure 10 clearly indicates that all energies are nearly in phase, in strong contrast to the polar fluxes shown in Figure 3. If anything, the flux changes at the higher energies slightly lead the those at the lower energies, a demonstration of the expected time dispersion for ENAs originating from a common source region. We conclude that since the phase differences are only seen at high latitudes and not low latitudes, they are due to the changes in the high-latitude energy distribution, brought about by the opening and closing of the PCHs.

Based on the results shown here and in the previous section, we find strong support for the notion that most polar ENAs observed by $I B E X$ at different energies are all from a common source region, namely the IHS. In addition, the correlation between the 1 au SW dynamic pressure and the ENA flux variation observed in the $I B E X$-Hi energy range is due to a combination of pressure balance and the propagation of structure along streamlines relatively close to the poles.

\section{SUMMARY}

With seven years of ENA observations from IBEX, the influence of the solar cycle on the polar ENA flux is becoming manifest. At both poles, the heliospheric ENA flux shows dramatic variations at all IBEX-Hi energies. The flux varies typically by a factor of two, and in the case of the highestenergy channel $(4.3 \mathrm{keV})$ by as much as a factor of four. The observation period is now long enough that the phasing of the flux variation is apparent. The lower energies show an initial decline followed by the beginnings of recovery, and the higher energies start relatively flat, or even go through a slight initial 


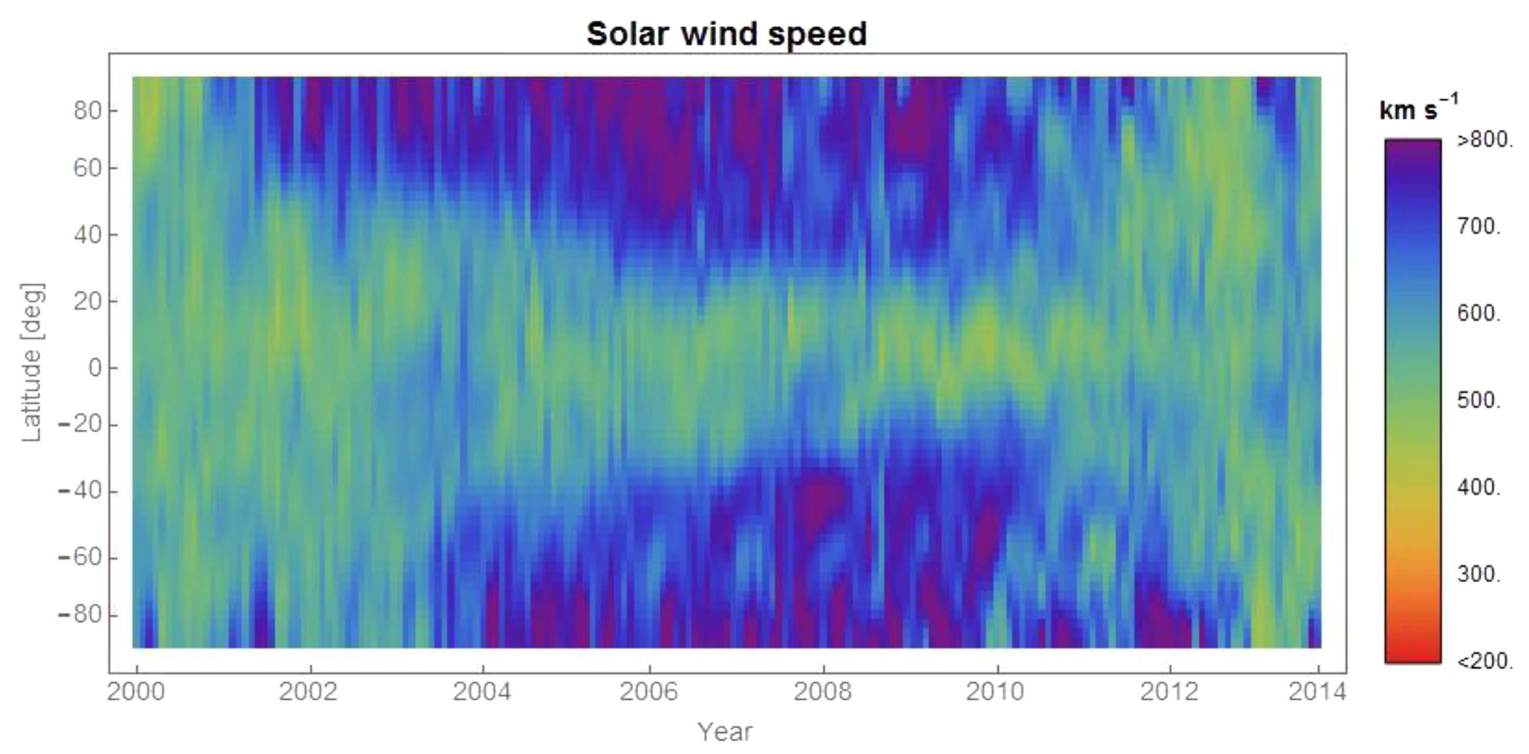

Figure 9. Heliolatitude vs. time map of the SW speed reconstructed from interplanetary scintillation (IPS) data and OMNI data, as described in Sokół et al. (2015).

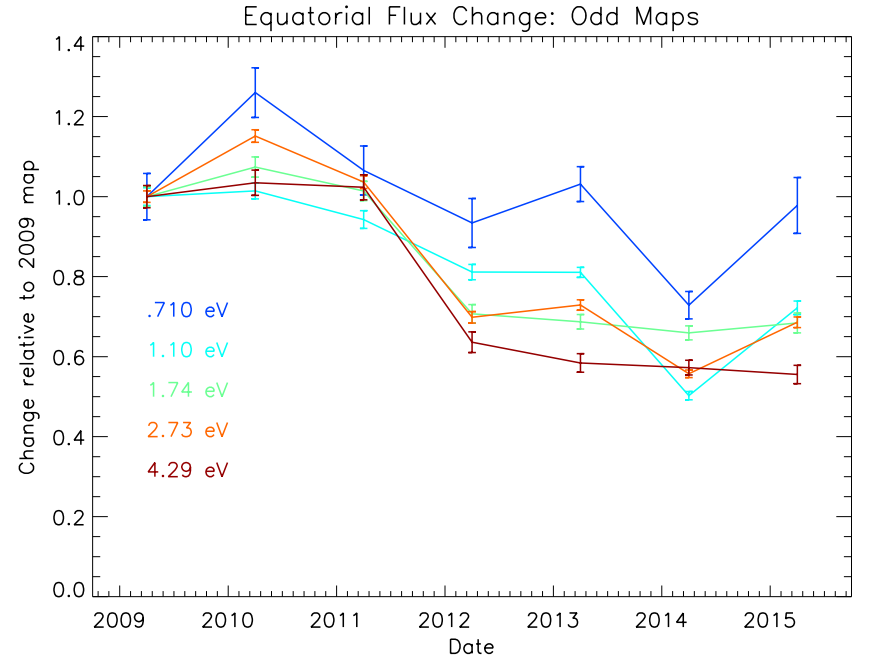

Figure 10. IBEX-Hi differential ENA energy fluxes at the ecliptic equator, normalized to the beginning of the mission. Points give the fluxes integrated over latitudes between $\pm 25^{\circ}$ of the equator and longitudes between $\pm 90^{\circ}$ of the heliospheric nose. Data from only the "odd" maps are used (that is, from the first six months of a given year) so as to use data with the same viewing configuration. The integration does not include pixels that include the Ribbon. These were removed using the mask derived by Schwadron et al. (2014).

rise, followed by a steady decrease that has yet to show any clear sign of slowing.

The ENA flux is a proxy for the plasma pressure within the IHS; thus, changes in the ENA flux are measures of changes in the heliosheath pressure. We argue that variations in the total heliosheath pressure are likely caused by variations in the SW dynamic pressure at the TS that then propagate through the IHS as pressure waves.

This is supported by the observation that the total LOSintegrated IHS pressure $\left(P_{\mathrm{s}} \cdot L\right)$ derived from the ENA flux at the north and south poles is well correlated with the SW dynamic pressure observed at $1 \mathrm{au}\left(P_{1 \mathrm{au}}\right)$ between late 2005 and late 2010 (for the north) and between early 2006 and the end of 2011 (for the south).
The correlation method allows us to estimate distances to the approximate center of the ENA source region, $220 \mathrm{au}$ in the direction of the north ecliptic pole and $190 \mathrm{au}$ in the direction of the south ecliptic pole. In addition, we can estimate the thickness of the IHS by combining these distances with the assumed distances to the TS at the poles using the TS distances measured by Voyagers 1 and 2 and scaling these to the poles based on published models of the outer heliosphere. From this, we arrive at estimates for the TS distance of $\sim 130 \mathrm{au}$ and $\sim 110$ au for the north and south pole directions, which leads us to estimates for the IHS thickness of $\sim 210 \mathrm{au}$ and $\sim 160 \mathrm{au}$, respectively. We note that these dimensions are larger than what are predicted by most (but not all) heliosphere models, and they are different from the observed heliosheath thickness in the Voyager 1 direction of $28 \mathrm{au}$. However, they are consistent with the measurements of Galli et al. (2016).

We further make the case that although the total IHS pressure is governed by the pressure balance across the TS, the ENA flux at a specific energy may still retain an imprint of the pre-TS conditions of the solar wind flowing along the streamlines from which those ENAs originate. Because of the large spatial size of the ENA-originating region and the range in travel times of heliosheath ions from various latitudes to the poles, the timescale for resolvable SW variability will be of order of a year or greater. Thus, individual SW transients will not be discernable (although the accumulated effect of multiple events may be). Rather, we expect primarily to see variations associated with the solar cycle itself. In addition, we argue that because of the finite lifetime of ions in the heliosheath, ENAs observed from the poles will predominately originate from IHS plasma flowing along streamlines originating as solar wind propagating outward at relatively high latitudes.

The evidence we see for this sort of ENA variation is that the flux in the highest-energy channel $(4.3 \mathrm{keV})$ closely tracks the areal extent of the polar coronal holes, clearly linking these ENAs to the very fast $\left(>700 \mathrm{~km} \mathrm{~s}^{-1}\right)$ solar wind emanating from these regions. This also explains the phase difference between the low-energy and high-energy ENAs. The lowenergy ENA flux varies with changes in the SW dynamic pressure over the course of the solar cycle, which is generally in phase with the sunspot number, whereas the high-energy 
flux cycles with the size of the PCHs, which is out of phase with the sunspot number.

We plan two avenues for further investigation. First, there is the straightforward continuation of the present work. We are within four to five years of collecting a complete solar cycle of ENA data, which will allow us to see the full evolutionary cycle of the polar ENA flux. In particular, we anticipate the recovery of the high-energy flux (ESAs 5 and 6) over the next few years.

Second, we plan to apply the pressure balance method for finding the dimensions of the heliosphere in the poleward directions to include ENA flux from the full energy range that contributes to the IHS pressure. Namely, we will include contributions derived from the ENAs observed by IBEX-Lo (Galli et al. 2016) and by Cassini/INCA (Krimigis et al. 2009). This avenue looks promising, as we already see there is spectral convergence (within error bars) between IBEX-Lo and IBEX-Hi across the overlapping $0.7-2 \mathrm{keV}$ energy range (see Figure 7, Galli et al. 2016). The IBEX-Hi and INCA energy ranges do not overlap, and there appears to be a spectral break at $\sim 6 \mathrm{keV}$, the boundary in their ranges (compare Figure 8 in Dialynas et al. 2013 with Figure 17 in Schwadron et al. 2011). However, the GDF sky maps at the highest $I B E X$-Hi channel $(4.7 \mathrm{keV})$ and the lowest INCA energy channel (5-13 keV) show a consistent structure across the sky (Dialynas et al. 2013).

Although we will not have the time resolution needed to accurately apply the correlation method, we can apply the technique first used in R2012, which employs the SW propagation model of Schwadron et al. (2011) to propagate the SW to the TS. Then, by requiring pressure balance across the TS, we can estimate the plasma pressure in the heliosheath and compare it to the LOS-integrated pressure derived from the ENA flux, which allows us to extract the LOS length $L$ from the ENA-derived $P \cdot L$.

We give our sincere thanks to all of the outstanding professionals who have made the IBEX mission a success. We would also like to thank Dr. John Steinberg for productive conversations regarding the latitude dependence of the ENA energy spectra, and the reviewer for very substantive and thoughtful comments. This work was carried out as part of NASA's IBEX Mission, with support from NASA's Explorer Program. Work at Los Alamos was performed under the auspices of the US Department of Energy. M.B., M.A.K. and J.M.S. acknowledge the support by the grant 2015-19-B-ST901328 from the National Science Center, Poland. N.K. is supported by the Schlumberger Foundation Faculty for the Future Program.

\section{REFERENCES}

Allegrini, F., Bzowski, M., Dayeh, M. A., et al. 2012, ApJL, 749, L41 Barnes, A. 1990, in Proc. COSPAR Coll. 1, Physics of the Outer Heliosphere, ed. S. Grzedzielski \& D. E. Page (Oxford: Permagon), 235
Belcher, J. W., Lazarus, A. J., McNutt, R. L., Jr., \& Gordon, G. S., Jr. 1993, JGR, 98, 15177

Burlaga, L. F., Ness, N. F., Acuna, M. H., et al. 2005, Sci, 309, 2027

Burlaga, L. F., Ness, N. F., Acuna, M. H., et al. 2009, ApJ, 692, 1125

Bzowski, M. 2008, A\&A, 488, 1057

Dayeh, M. A., McComas, D. J., Allegrini, F., et al. 2012, ApJ, 749, 50

Dayeh, M. A., McComas, D. J., Livadiotis, G., et al. 2011, ApJ, 734, 29

Desai, M. I., Allegrini, F., Dayeh, M. A., et al. 2015, ApJ, 802, 100

Dialynas, K., Krimigis, S. M., Mitchell, D. G., et al. 2015, JPhCS, 577, 012007

Dialynas, K., Krimigs, S. M., Mitchell, D. G., et al. 2013, ApJ, 778, 40

Ebert, R. W., McComas, D. J., Elliott, H. A., et al. 2009, JGR, 114, A01109

Fahr, H.-J., \& Scherer, K. 2004, ASTRA, 1, 3

Florinski, V., Zank, G. P., \& Pogorelov, N. V. 2005, JGR, 110, A07104

Funsten, H. O., Allegrini, F., Bochsler, P., et al. 2009a, SSRv, 146, 75

Funsten, H. O., Allegrini, F., Crew, G. B., et al. 2009b, Sci, 326, 964

Funsten, H. O., Harper, R. W., \& McComas, D. J. 2005, RScI, 76, 053301

Fuselier, S. A., Allegrini, F., Bzowski, M., et al. 2012, ApJ, 754, 14

Fuselier, S. A., Allegrini, F., Funsten, H. O., et al. 2009a, Sci, 326, 962

Fuselier, S. A., Bochsler, P., Chornay, D., et al. 2009b, SSRv, 146, 117

Galli, A., Wurz, P., Fuselier, S. A., et al. 2014, ApJ, 796, 9

Galli, A., Wurz, P., Schwadron, N. A., et al. 2016, ApJ, 821, 107

Izmodenov, V. V., Malama, Y. G., \& Ruderman, M. S. 2008, AdSpR, 41, 318

Izmodenov, V. V., Malama, Y. G., Ruderman, M. S., et al. 2009, SSRv, 145,329

Karna, N., Hess Webber, S. A., \& Pesnell, W. D. 2014, SoPh, 289, 3381

King, J. H., \& Papitashvili, N. E. 2005, JGR, 110, A02104

Krimigis, S. M., Mitchell, D. G., Roelof, E. C., et al. 2009, Sci, 326, 971

Krimigis, S. M., Roelof, E. C., Decker, R. B., et al. 2011, Natur, 474, 359

Lazarus, A. J., \& McNutt, R. L., Jr. 1990, in Physics of the Outer Heliosphere, ed. S. Grzedzielski \& D. E. Page (Oxford: Pergamon), 229

Lindsay, B. G., \& Stebbings, R. F. 2005, JGR, 110, A12213

McComas, D. J., Allegrini, F., Bochsler, P., et al. 2009a, SSRv, 1436, 11

McComas, D. J., Allegrini, F., Bochsler, P., et al. 2009b, Sci, 326, 959

McComas, D. J., Allegrini, F., Bzowski, M., et al. 2014, ApJS, 213, 20

McComas, D. J., Barraclough, B. L., Funsten, H. O., et al. 2000, JGR, 105, 10,419

McComas, D. J., Bzowski, M., Frisch, P., et al. 2010, JGR, 115, A09113

McComas, D. J., Bzowski, M., Fuselier, S. A., et al. 2015, ApJS, 220, 22

McComas, D. J., Carrico, J. P., Hautamaki, B., et al. 2011, SpWea, 9, S11002

McComas, D. J., Dayeh, M. A., Allegrini, F., et al. 2012, ApJS, 203, 1

McComas, D. J., Ebert, R. W., Elliott, H. A., et al. 2008, GRL, 35, L18103

McComas, D. J., \& Schwadron, N. A. 2014, ApJL, 795, L17

Möbius, E., Bochsler, P., Bzowski, M., et al. 2012, ApJS, 198, 11

Opher, M., Drake, J. F., Zieger, B., \& Gombosi, T. I. 2015, ApJL, 800, L28

Opher, M., Richardson, J. D., Toth, G., et al. 2009, SSRv, 143, 43

Pogorelov, N. V., Seuss, S. T., Borovikov, S. N., et al. 2013, ApJ, 772, 2

Pogorelov, N. V., Stone, E. C., Florinski, V., et al. 2007, ApJ, 668, 611

Reisenfeld, D. B., Allegrini, F., Bzowski, M., et al. 2012, ApJ, 747, 110

Roelof, E. C., Krimigis, S. M., Mitchell, D. G., et al. 2012, in AIP Conf. Proc.

1436, Physics of the Heliosphere: A 10 Year Retrospective, ed.

J. Heerikhuisen et al. (Melville, NY: AIP), 239

Schwadron, N. A., Allegrini, F., Bzowski, M., et al. 2011, ApJ, 731, 56

Schwadron, N. A., Bzowski, M., Crew, G. B., et al. 2009, Sci, 326, 966

Schwadron, N. A., Möbius, E., Fuselier, S. A., et al. 2014, ApJS, 215, 13

Siewert, M., Fahr, H.-J., \& McComas, D. J. 2014, A\&A, 565, A81

Sokół, J. M., Bzowski, M., Tokumaru, M., et al. 2013, SoPh, 285, 167

Sokół, J. M., Swaczyna, P., Bzowski, M., et al. 2015, SoPh, 290, 2589

Sternal, O., Fichtner, H., \& Scherer, K. 2008, A\&A, 477, 365

Stone, E. C., Cummings, A. C., McDonald, F. B., et al. 2008, Natur, 454, 71

Stone, E. C., Cummings, A. C., McDonald, F. B., et al. 2013, Sci, 341, 150

Tokumaru, M., Kojima, M., \& Fujiki, K. 2012, JGR, 117, A06108

Washimi, H., Zank, G. P., Hu, Q., et al. 2011, MNRAS, 416, 1475

Wurz, P., Fuselier, S. A., Möbius, E., et al. 2009, SSRv, 146, 173

Zirnstein, E. J., Heerikhuisen, J., Pogorelov, N. V., et al. 2015, ApJ, 804, 5 